\title{
Magnetic-free traveling-wave nonreciprocal superconducting microwave components
}

\author{
Dengke Zhang ${ }^{1,2}$, and Jaw-Shen Tsai ${ }^{1,2, *}$ \\ ${ }^{1}$ Department of Physics, Tokyo University of Science, Kagurazaka, Tokyo 162-8601, Japan \\ ${ }^{2}$ RIKEN Center for Quantum Computing, RIKEN, Wako, Saitama 351-0198, Japan
}

\begin{abstract}
We propose a design to realize integrated broadband nonreciprocal microwave isolators and circulators using superconducting circuit elements without any magnetic materials. To obtain a broadband response, we develop a waveguide-based design by temporal modulations. The corresponding compact traveling-wave structure is implemented with integrated superconducting composite right-/left-handed transmission lines. The calculations show that the bandwidth of $580 \mathrm{MHz}$ can be realized over a nonreciprocal isolation of $20 \mathrm{~dB}$ in reflections. Such on-chip isolators and circulators are useful for cryogenic integrated microwave connections and measurements, such as protecting qubits from the amplified reflected signal in multiplexed readout.
\end{abstract}

\section{INTRODUCTION}

Realizing on-chip nonreciprocal isolation is a significant difficulty in microwave integrated circuits [1-3]. The need to overcome this difficulty has recently become increasingly urgent for on-chip microwave quantum information processing, especially for low-noise quantum measurements [4-18]. Achieving nonreciprocity requires breaking time-reversal symmetry, which can be accomplished via the magneto-optical effect, nonlinearity, or spatiotemporal modulation [1, 2, 12, 19, 20]. Although each one has its own advantages, on-chip integration remains a huge challenge because the reported implementations so far suffer from high complexity, intricate control, small bandwidth, or large footprint. Achieving the magnetooptical effect requires magneto-optical materials, such as ferrites, which are difficult to be integrated by the standard process and unavoidably introduce unwanted magnetic noise [21, 22]. Nonreciprocity using nonlinearity is power-dependent, which means that the nonreciprocal performance depends on input power; furthermore, some reciprocal leakages happen with multi-inputs [23-25]. In recent years, the method of spatiotemporal modulation has shown powerful vitality because diverse modulations can be provided by electric, acoustic, or mechanical dynamic biasing [1, 2, 12, 26-34].

In general, the spatiotemporal modulation requires simultaneously spatially and temporally dynamic modulations of the material parameters, such as the refractive index [19, 26, 29, 35]. In resonance-based configurations, such modulations are implemented by applying spatially uniform modulation but specific timing control on each resonator or coupling segment [12, 13, 36-38]. The complexities of these types of structure and controls are partly eased at the expense of working bandwidth. To obtain a broadband nonreciprocal response, waveguide-based implementations are needed. Common spatiotemporal modulation for a waveguide is very difficult for fabricating the structure or applying the modulation [19, 35, 39].

\footnotetext{
*tsai@riken.jp
}

Specifically, such a spatiotemporal modulation can also be achieved by a strong microwave field for a microwave isolator or circulator. Ranzani et al. have experimentally demonstrated a broadband isolator using a Josephsonjunction transmission line [40]. In their scheme, an intraband transition was achieved by spatiotemporal modulation with a microwave pumping. As the frequency of pumping (or modulation) is very high (comparable to the frequency of signal), it is not easy to well terminate both modes simultaneously, the frequencies of which are spaced by a value of the modulation frequency.

Recently, a proposal has suggested that a broadband nonreciprocal isolation can be realized by applying two spatially uniform modulations on two waveguide-based mode transformers, in which the interband transition between two modes is required by means of a dynamic modulation [41, 42]. With such an approach, the requirement of spatiotemporal modulation is reduced to two spatially discrete temporal-modulations. Thus, the research priority becomes how to elegantly realize a temporally modulated mode transformer with waveguides. Moreover, the termination impedance matching for both modes are required to eliminate the reflections. In a superconducting circuit, the couplings between two common coplanar waveguides (CPWs) are very weak, which makes it difficult to achieve the required mode transformation within a small footprint. In addition, the interband transition cannot be realized by a simple modulation of coupled CPWs. Here, we propose a new design of integrated microwave isolator/circulator with superconducting composite right/left-handed (CRLH) transmission lines (TLs) [43, 44]. There are several merits to using CRLH TLs: First, the supported light possesses a small effective wavelength, which means that a strong coupling can be realized between such two TLs and, meanwhile, a small footprint can also be achieved. Second, the coupled CRLH TLs can be modulated in a low frequency, which implies the frequency gap of coupled two modes is small. This feature will be a benefit to realizing a successful termination impedance matching for both modes. Third, CRLH TLs are one kind of metamaterials-based waveguides that holds a high design degree of freedom. 
In this work, we design a structure for realizing the broadband nonreciprocal isolation with the CRLH TLs. In the designed structure, the required mode transformation is accomplished with two coupled CRLH TLs. The entire designed structure can be fabricated with a standard superconducting circuit process, and the spatially uniform modulations are readily applied using electric local biasing lines. This paper is organized as follows. In Section II, we present the structure design and the corresponding model, and an intuitive operation principle is given with the help of Ramsey interference. In Section III, we introduce the CRLH TLs and their couplings. Then a 3-dB CRLH directional coupler is implemented and modulated, as described in Section IV. Section V shows the nonreciprocal responses of the entire designed CRLH-based structure and Section VI provides a possible design layout with superconducting lines and Josephson junctions, and some discussions about the improvement. Last, we present the conclusion in Section VII.

\section{STRUCTURE DESIGN AND MODEL}

The proposed structure contains three stages: a 3$\mathrm{dB}$ forward-wave directional coupler (stage-I: plane- $\mathcal{A}$ to plane- $\mathcal{B})$, then a phase difference between the two split beams introduced through two uncoupled waveguides (stage-II: plane- $\mathcal{B}$ to plane- $\mathcal{C}$ ), and finally followed by another 3 -dB forward-wave directional coupler (stage-III: plane- $\mathcal{C}$ to plane- $\mathcal{D}$ ), as shown in Fig. 1(a). Importantly, the two 3-dB forward-wave directional couplers have a time-varying coupling coefficient via dynamic modulation. As shown in Fig. 1(a), we consider that each coupler consists of two waveguides, whose eigenmodes are mode- $[\uparrow]$ and mode- $[\downarrow]$ propagating along the $z$-direction, respectively. Assume that the time-varying coupling coefficient between mode- $[\uparrow]$ and mode- $[\downarrow]$ is expressed by

$$
K(t)=K_{0} \cos \left(\Omega_{\mathrm{m}} t+\phi\right),
$$

where $K_{0}$ is the maximum coupling coefficient (i.e., the amplitude of $K), \Omega_{\mathrm{m}}$ is the modulation frequency, and $\phi$ is the modulation initial phase. In this work, the 3 - $\mathrm{dB}$ beam coupling means that there is a relation of $\left|K_{0} l_{c}\right|=\pi / 4$ with coupling length $l_{\mathrm{c}}$ of the coupler. In the frame rotating with the uncoupled waveguides' propagation constants, the equation of motion of fields in the directional coupler can be written with rotating wave approximation (see Appendix A for the details) as

$$
\mathrm{j} \frac{\partial}{\partial z}\left[\begin{array}{l}
a_{\uparrow} \\
a_{\downarrow}
\end{array}\right]=\left[\begin{array}{cc}
0 & K_{0} \mathrm{e}^{-\mathrm{j} \phi} \\
K_{0} \mathrm{e}^{\mathrm{j} \phi} & 0
\end{array}\right]\left[\begin{array}{l}
a_{\uparrow} \\
a_{\downarrow}
\end{array}\right]
$$

where $a_{\uparrow}$ and $a_{\downarrow}$ represent field amplitudes of mode- $[\uparrow]$ and mode- $[\downarrow]$, respectively [41, 45]. By solving Eq. (2), we can deduce the spatial evolutions of mode- $[\uparrow]$ and mode- $[\downarrow]$ along the $z$-direction
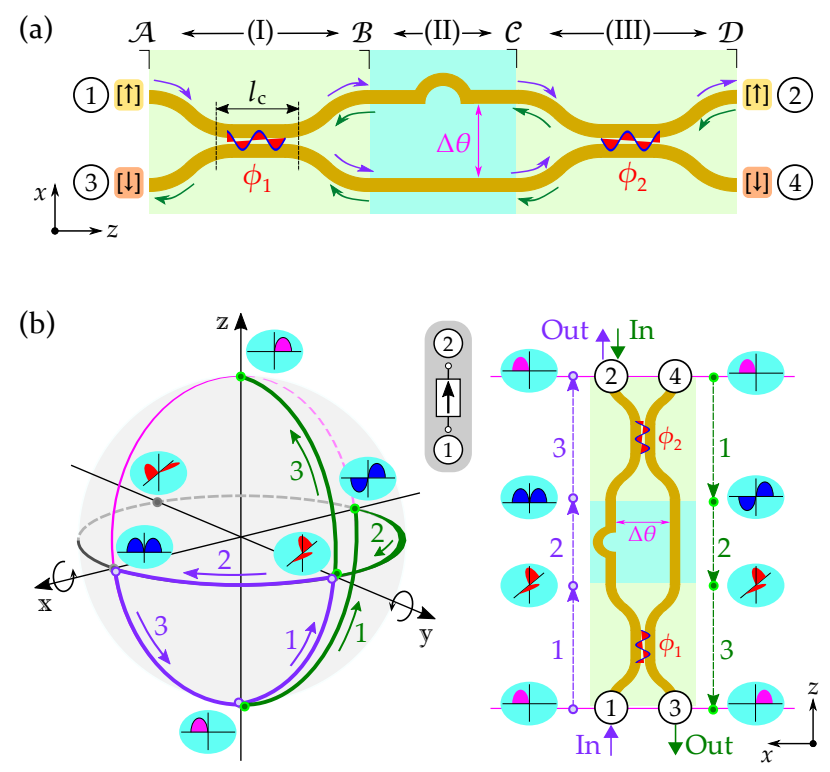

Figure 1. Schematic of the design and working principle. (a) The designed structure is constructed with a cascade of three stages of dual-waveguide configuration, where the two bare waveguides support guided mode- $[\uparrow]$ and mode- $[\downarrow]$, respectively. Stage-I and stage-III are 3-dB forward-wave directional couplers, the coupling coefficients of which are temporally modulated with initial phases of $\phi_{1}$ and $\phi_{2}$, respectively. Stage-II is two uncoupled waveguides with different propagation lengths to introduce a phase difference of $\Delta \theta$. The entire structure performs as a four-port network, which is indexed by port-1 to port-4. (b) Field evolutions are displayed on a Bloch sphere (left) and in real space (right) with different injections at port-1 and port- 2 . Here, we set $\left\{\phi_{1}, \Delta \theta, \phi_{2}\right\}=\{0, \pi / 2, \pi / 2\}$. The light injected at port-1 will be output via port-2, whereas the input light at port-2 will go to port-3. These behaviors indicate that a nonreciprocal isolation between port-1 and port-2 can be achieved with the design shown in (a).

$\left[\begin{array}{c}a_{\uparrow}(z) \\ a_{\downarrow}(z)\end{array}\right]=\left[\begin{array}{cc}\cos \left(K_{0} z\right) & -\mathrm{je} \mathrm{e}^{-\mathrm{j} \phi} \sin \left(K_{0} z\right) \\ -\mathrm{j} \mathrm{j}^{\mathrm{j} \phi} \sin \left(K_{0} z\right) & \cos \left(K_{0} z\right)\end{array}\right]\left[\begin{array}{c}a_{\uparrow}(0) \\ a_{\downarrow}(0)\end{array}\right]$.

According to the definition of the scattering matrix of a two-port network, the scattering matrix $\left[\mathbf{M}^{(\mathrm{c})}\right]$ of the directional coupler can be obtained straightforwardly through Eq. (3). For the 3-dB coupler in stage-I, using $K_{0} l_{\mathrm{c}}=\pi / 4$, we can express the scattering matrix (relation of fields between ports in plane- $\mathcal{A}$ and ports in plane- $\mathcal{B})$ as

$$
\mathbf{M}^{(\mathrm{c})}\left(\phi_{1}\right)=\frac{1}{\sqrt{2}}\left[\begin{array}{cc}
1 & -\mathrm{je} \mathrm{e}^{-\mathrm{j} \phi_{1}} \\
-\mathrm{je} \mathrm{e}^{\mathrm{j} \phi_{1}} & 1
\end{array}\right],
$$

where $\phi_{1}$ is the modulation initial phase of the $3-\mathrm{dB}$ coupler in stage-I. In comparison with the scattering matrix of a common (unmodulated) 3-dB directional coupler, we can find that a phase shift $\phi_{1}$ is appended to the cross-coupling parameter of the modulated coupler (i.e., 
transition between mode- $[\uparrow]$ and mode- $[\downarrow])$, resulting from the dynamic modulation of the coupling coefficient. Regarding the coupler in stage-III (from plane- $\mathcal{C}$ to plane- $\mathcal{D}$ ), the corresponding scattering matrix $\mathbf{M}^{(\mathrm{c})}\left(\phi_{2}\right)$ can be readily given with the replacement of $\phi_{1}$ by $\phi_{2}$ in Eq. (4). For the two uncoupled waveguides in stage-II, the scattering matrix can be represented by

$$
\mathbf{M}^{(\mathrm{b})}(\Delta \theta)=\left[\begin{array}{cc}
\mathrm{e}^{\mathrm{j} \Delta \theta} & 0 \\
0 & 1
\end{array}\right]
$$

where $\Delta \theta$ is the introduced phase difference between propagating waves of mode- $[\uparrow]$ and mode- $[\downarrow]$.

By using a cascade of the scattering matrices of the three stages, we can easily obtain the scattering matrix of the entire structure. For the forward ( $+z$-direction) propagation waves, the scattering matrix is written as

$$
\mathbf{M}^{(\Rightarrow)}=\mathbf{M}^{(\mathrm{c})}\left(\phi_{2}\right) \mathbf{M}^{(\mathrm{b})}(\Delta \theta) \mathbf{M}^{(\mathrm{c})}\left(\phi_{1}\right),
$$

and for the backward (-z-direction) propagation waves, the corresponding scattering matrix is expressed as

$$
\mathbf{M}^{(\Leftarrow)}=\mathbf{M}^{(\mathrm{c})}\left(\phi_{1}\right) \mathbf{M}^{(\mathrm{b})}(\Delta \theta) \mathbf{M}^{(\mathrm{c})}\left(\phi_{2}\right) .
$$

Here, we assume that $\left\{\phi_{1}, \Delta \theta, \phi_{2}\right\}=\{0, \pi / 2, \pi / 2\}$; the two specific scattering matrices are calculated as

$$
\mathbf{M}^{(\Rightarrow)}=\left[\begin{array}{ll}
\mathrm{j} & 0 \\
0 & 1
\end{array}\right], \quad \mathbf{M}^{(\Leftarrow)}=\left[\begin{array}{cc}
0 & -\mathrm{j} \\
1 & 0
\end{array}\right] .
$$

From Eq. (8), we can find that a forward injected field of mode- $[\uparrow]$ can be maintained finally, whereas a backward injected field of mode- $[\uparrow]$ is transferred to the field of mode- $[\downarrow]$, which are shown by paths with purple and green arrows in Fig. 1(a), respectively. That is, the transmission of light from port-1 to port- 2 is allowed, but the reverse process is suppressed (the input light at port- 1 goes to port-3). Such a behavior implies that a nonreciprocal isolation can be achieved using our design with the specific settings for $\left\{\phi_{1}, \Delta \theta, \phi_{2}\right\}$.

As a dual-mode system, a geometric description will be useful for understanding the working principle [46-48]. For our structure, the processes of mode transformations can be described by a photonic Ramsey interference. The function of a $3-\mathrm{dB}$ directional coupler is to apply a " $\pi / 2$ pulse" excitation to the input field of mode- $[\uparrow]$ or mode- $[\downarrow]$. After passing through the $3-\mathrm{dB}$ directional coupler, the input field will be transferred to a superposition field of mode- $[\uparrow]$ and mode- $[\downarrow]$. This means that the mode transformation is achieved by the $3-\mathrm{dB}$ directional coupler. Stage-II works like a free evolution of the superposition state after a detuned $\pi / 2$-pulse driving, which is realized by introducing a phase difference between field components of mode- $[\uparrow]$ and mode- $[\downarrow]$. Finally, another $\pi / 2$-pulse is applied by the 3 - $\mathrm{dB}$ directional coupler in
stage-III. In particular, the modulation initial phases $\left(\phi_{1}\right.$ and $\phi_{2}$ ) applied in the couplers play the role of the initial phase of a $\pi / 2$-pulse signal. For the special setting of $\left\{\phi_{1}, \Delta \theta, \phi_{2}\right\}=\{0, \pi / 2, \pi / 2\}$, the field evolutions on a Bloch (or Poincaré) sphere and in real space are displayed in Fig. 1(b). The forward evolution with the input field at port- 1 is represented by purple curves, and the backward evolution with the input field at port-2 is denoted by green curves. These demonstrations clearly show that the isolation response between port- 1 and port- 2 is realized by performing such a three-stage spatial operation.

To implement such a structure in a superconducting circuit, a real 3-dB directional coupler is needed; moreover, the coupling coefficient can be modulated. However, it is unrealistic to achieve an on-chip 3-dB directional coupler using common CPWs, because the coupling between two parallel superconducting CPWs is very weak. To realize such a compact 3-dB directional coupler on a chip, CRLH TLs are introduced in this work.

\section{CRLH TRANSMISSION LINES}

(a)
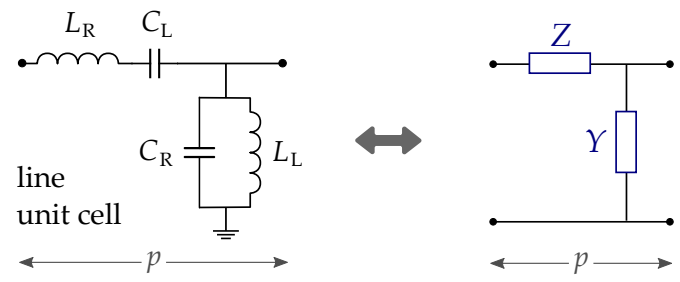

(b)

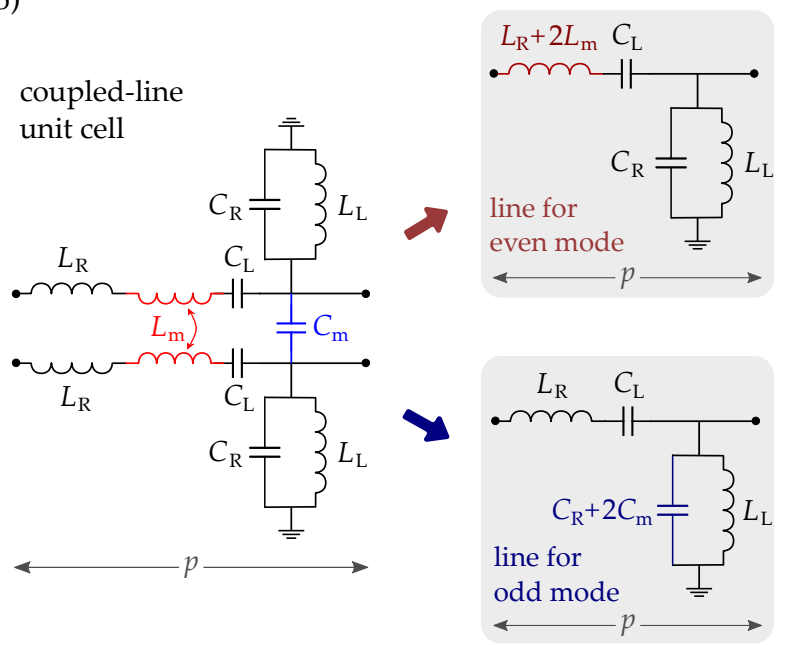

Figure 2. Unit cells of CRLH TL and coupled-line. (a) Circuit model of a unit cell of CRLH TL. (b) Circuit model of unit cells of the CRLH directional coupler and the corresponding evenand odd-mode TL models. The even- and odd-mode equivalent circuits have the same topology but with different substitutions $L_{\mathrm{R}} \rightarrow L_{\mathrm{R}}+2 L_{\mathrm{m}}$ and $C_{\mathrm{R}} \rightarrow C_{\mathrm{R}}+2 C_{\mathrm{m}}$, respectively. 


\section{A. Periodic CRLH TLs}

Here, we use the CRLH TLs to realize a compact broadband forward-wave directional coupler. The reason is that there are two benefits with CRLH TLs: first, the wavenumber of guided modes in CRLH TLs can be very large, which means that the effective wavelength is very small; second, the strong coupling between such two CRLH TLs can be realized by introducing optimal mutual inductance or capacitance [43]. In reality, an equivalent CRLH TL is constructed by periodically repeating a small lumped unit cell with pitch $p$, as shown in Fig. 2(a). The unit cell consists of the combination of right-handed elements of series inductance $L_{\mathrm{R}}$ and shunt capacitance $C_{\mathrm{R}}$ with left-handed elements of shunt inductance $L_{\mathrm{L}}$ and series capacitance $C_{\mathrm{L}}$ (see Refs. [43, 49, 50]). For simplicity, considering the lossless TLs, the propagation constant $\beta$ satisfies the relation $\beta=-\mathrm{j} \gamma$, where $\gamma$ is the complex propagation constant. According to the Bloch-Floquet theorem, the dispersion relation of a CRLH TL can be obtained by applying periodic boundary conditions to a unit cell in the form

$$
\beta(\omega)=\frac{1}{p} \cos ^{-1}\left[1+\frac{Z(\omega) Y(\omega)}{2}\right],
$$

where the series impedance $(Z)$ and shunt admittance $(Y)$ of the unit cell are respectively given by

$$
\begin{aligned}
& Z(\omega)=\mathrm{j}\left(\omega L_{\mathrm{R}}-\frac{1}{\omega C_{\mathrm{L}}}\right), \\
& Y(\omega)=\mathrm{j}\left(\omega C_{\mathrm{R}}-\frac{1}{\omega L_{\mathrm{L}}}\right) .
\end{aligned}
$$

Furthermore, the characteristic impedance $\left(Z_{0}\right)$ of a CRLH TL is read as

$$
Z_{0}(\omega)=\sqrt{Z(\omega) / Y(\omega)}
$$

As a comparison demonstrated in Appendix B, a homogeneous TL with $\beta, Z_{0}$ can effectively model the periodic CRLH TL in the long-wavelength limit.

\section{B. Coupling of two identical CRLH TLs}

A CRLH directional coupler can be constructed by introducing mutual inductance $L_{\mathrm{m}}$ or capacitance $C_{\mathrm{m}}$ into two CRLH TLs, as shown in Fig. 2(b). Here, we consider a symmetric directional coupler, where the two bare CRLH TLs are identical. In a coupled-line, the two bare guided modes are mixed and generate two hybrid guided modeseven-mode and odd-mode. The two normal modes can be analytically reduced to guided modes of two different bare CRLH TLs. As displayed in Fig. 2(b), the two effective CRLH TLs for even and odd modes have the same topology as that of the initial bare CRLH TLs [44, 51]. The difference between the two effective CRLH TLs is
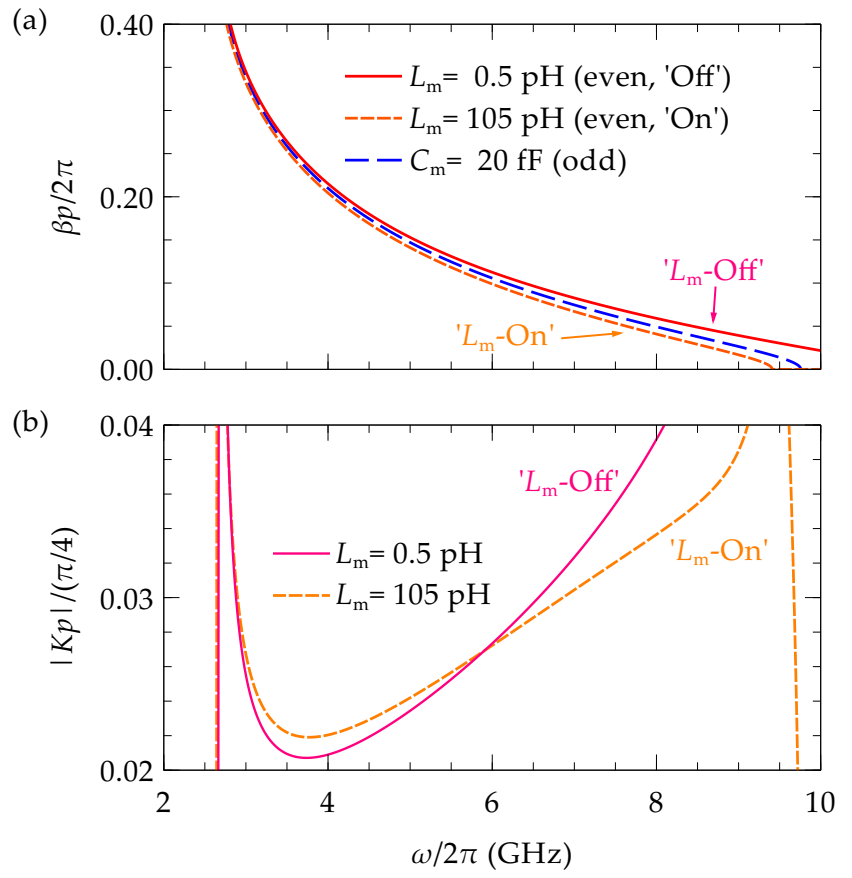

Figure 3. Dispersion relations and coupling coefficients. (a) Dispersion curves for odd-mode with $C_{\mathrm{m}}=20 \mathrm{fF}$ and evenmode with $L_{\mathrm{m}}=0.5 \mathrm{pH}$ and $105 \mathrm{pH}$. The lower $L_{\mathrm{m}}$ is called ' $L_{\mathrm{m}}$-Off' and the upper $L_{\mathrm{m}}$ is called ' $L_{\mathrm{m}}$-On'. (b) Frequency dependence of magnitudes of coupling coefficients under the conditions of ' $L_{\mathrm{m}}$-Off' and ' $L_{\mathrm{m}}$-On', in which the odd-mode is fixed with $C_{\mathrm{m}}=20 \mathrm{fF}$ in the calculations.

$L_{\mathrm{R}}$ revised by the addition of $2 L_{\mathrm{m}}$ for the even-mode and $C_{\mathrm{R}}$ revised by the addition of $2 C_{\mathrm{m}}$ for the odd-mode. As the topologies of even/odd lines are the same as that of a bare CRLH TL, the analyses of the CRLH TL can be applied directly to calculate the corresponding parameters of even and odd modes with appropriate substitutions in Eqs. (9-12). We use the notation $\beta_{\text {even }}, Z_{\text {even }}, Y_{\text {even }}$, and $Z_{0 \text { even }}$ for the even-line and $\beta_{\text {odd }}, Z_{\text {odd }}, Y_{\text {odd }}$, and $Z_{0 \text { odd }}$ for the odd-line. According to the coupled-mode theory, the coupling coefficient of the coupler can be given by (see Appendix A for the details)

$$
K=\frac{\beta_{\text {even }}-\beta_{\text {odd }}}{2} .
$$

The equation tells us that the coupling coefficient can be modulated if either or both $\beta_{\text {even }}$ and $\beta_{\text {odd }}$ could be changed in a controllable manner. In this work, we will tune $\beta_{\text {even }}$ by varying the mutual inductance $L_{\mathrm{m}}$ to achieve the modulation of the coupling coefficient $K$. For the varied $L_{\mathrm{m}}$, we call the lower bound ' $L_{\mathrm{m}}$-Off' and the upper bound ' $L_{\mathrm{m}}$-On.' According to the modulation form of $K$ in (1), the two bound limits will exactly induce the extreme of $K$, that is, $K_{0}$ and $-K_{0}$, respectively. This also implies that both the $L_{\mathrm{m}}$-Off and $L_{\mathrm{m}}$-On will generate the 3 -dB coupling if $\left|K_{0} l_{\mathrm{c}}\right|=\pi / 4$.

In this work, we demonstrate microwave responses 
around $6 \mathrm{GHz}$, which is a typical frequency range for the superconducting circuits. Here, we set $\left\{L_{\mathrm{R}}, C_{\mathrm{R}}\right\}=$ $\{300 \mathrm{pH}, 150 \mathrm{fF}\}$ and $\left\{L_{\mathrm{L}}, C_{\mathrm{L}}\right\}=\{1400 \mathrm{pH}, 560 \mathrm{fF}\}$ for the bare CRLH TLs. Furthermore, to realize a 3 -dB coupler with an operating frequency of $6 \mathrm{GHz}, C_{\mathrm{m}}$ is fixed as $20 \mathrm{fF}$, and meanwhile $L_{\mathrm{m}}$ can take the value of $0.5 \mathrm{pH}$ or $105 \mathrm{pH}$, which correspond to $L_{\mathrm{m}}$-Off and $L_{\mathrm{m}}-\mathrm{On}$, respectively. By using Eq. (9), we can calculate the dispersion curve of the odd-mode with $C_{\mathrm{m}}=20 \mathrm{fF}$. Correspondingly, the dispersion curves of the even-mode with $L_{\mathrm{m}}$-Off and $L_{\mathrm{m}}-\mathrm{On}$ are calculated using $L_{\mathrm{m}}=0.5 \mathrm{pH}$ and $105 \mathrm{pH}$, respectively. These results are shown in Fig. 3(a) and indicate that the dispersion curve of the odd-mode is in the middle of that of the even-mode of $L_{\mathrm{m}}$-Off and $L_{\mathrm{m}}$-On. By using the expression of $K$ in Eq. (13), the magnitude of the coupling coefficient can be changed from maximum negative to maximum positive when the $L_{\mathrm{m}}$ is continuously tuned from $L_{\mathrm{m}}$-Off to $L_{\mathrm{m}}$-On. This means that modulation of the coupling coefficient as the form of Eq. (1) is possible if we could temporally vary the $L_{\mathrm{m}}$ between $L_{\mathrm{m}}$-Off and $L_{\mathrm{m}}$-On. Figure $3(\mathrm{~b})$ displays the magnitudes of extreme coupling coefficients with $L_{\mathrm{m}}$-Off and $L_{\mathrm{m}}$-On. As the design goal at the operating frequency of $6 \mathrm{GHz}$, the two extreme coupling coefficients have the same magnitude, which is just the maximum coupling coefficient $K_{0}$. This means that the same power-splitting performance can be realized for $L_{\mathrm{m}}$-Off and $L_{\mathrm{m}}$-On at $6 \mathrm{GHz}$. In Fig. 3(b), the two curves separate when the frequency deviates from $6 \mathrm{GHz}$, which results from the structural dispersions of different $L_{\mathrm{m}}$. It is clear that the $L_{\mathrm{m}}$-Off coupling coefficient experiences a stronger dispersion, which will bring a more severe restriction on the operating bandwidth.

\section{MODULATED DIRECTIONAL COUPLER}

\section{A. The 3-dB forward-wave coupler}

To obtain the response of coupled lines, a general approach with the $A B C D$-matrix for TLs is adopted [49, 51]. First, the $A B C D$-matrix of a unit cell (in a symmetric form) for an even-line is computed as

$$
\left[\begin{array}{cc}
A_{\mathrm{e}} & B_{\mathrm{e}} \\
C_{\mathrm{e}} & D_{\mathrm{e}}
\end{array}\right]=\left[\begin{array}{cc}
1+Z_{\text {even }} Y_{\text {even }} / 2 & Z_{\text {even }}+Z_{\text {even }}^{2} Y_{\text {even }} / 4 \\
Y_{\text {even }} & 1+Z_{\text {even }} Y_{\text {even }} / 2
\end{array}\right]
$$

Then, the $A B C D$-matrix of an $N$-cell even-line can be deduced

$$
\left[\begin{array}{ll}
A_{\mathrm{e} N} & B_{\mathrm{e} N} \\
C_{\mathrm{e} N} & D_{\mathrm{e} N}
\end{array}\right]=\left[\begin{array}{cc}
A_{\mathrm{e}} & B_{\mathrm{e}} \\
C_{\mathrm{e}} & D_{\mathrm{e}}
\end{array}\right]^{N}
$$

Once the $A B C D$-matrix for an $N$-cell TL has been established, the corresponding scattering matrix $\mathbf{S}^{[\mathrm{e}]}$ of the $N$-cell even-line for terminations of impedance $Z_{\text {c }}$ can be computed as
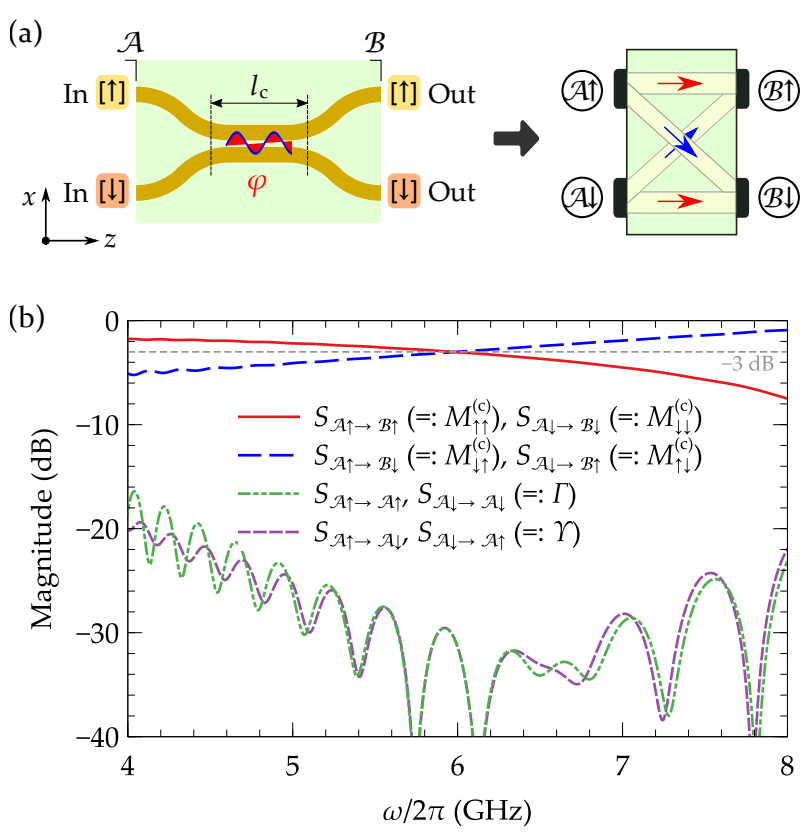

Figure 4. The 3-dB forward-wave directional coupler. (a) Sketch of a directional coupler and the reduced four-port network. (b) Scattering parameters of 3-dB coupler with $l_{\mathrm{c}}=37 p$ for operating frequency of $6 \mathrm{GHz}$. In the calculations, we adopt the same values of $\left\{L_{\mathrm{R}}, C_{\mathrm{R}}\right\}$ and $\left\{L_{\mathrm{L}}, C_{\mathrm{L}}\right\}$ as these used in Fig. 3(a) associated with $C_{\mathrm{m}}=20 \mathrm{fF}$ for the odd-mode and $L_{\mathrm{m}}=0.5 \mathrm{pH}$ (i.e., $L_{\mathrm{m}}$-Off) for the even-mode.

$$
\begin{aligned}
S_{\mathcal{A \mathcal { A }}}^{\mathrm{e}]} & =\left(A_{\mathrm{e} N}+B_{\mathrm{e} N} / Z_{\mathrm{c}}-C_{\mathrm{e} N} Z_{\mathrm{c}}-D_{\mathrm{e} N}\right) / E \\
S_{\mathcal{A B}}^{[\mathrm{e}]} & =2\left(A_{\mathrm{e} N} D_{\mathrm{e} N}-B_{\mathrm{e} N} C_{\mathrm{e} N}\right) / E \\
S_{\mathcal{B} \mathcal{A}}^{[\mathrm{e}]} & =2 / E \\
S_{\mathcal{B B}}^{[\mathrm{e}]} & =\left(-A_{\mathrm{e} N}+B_{\mathrm{e} N} / Z_{\mathrm{c}}-C_{\mathrm{e} N} Z_{\mathrm{c}}+D_{\mathrm{e} N}\right) / E
\end{aligned}
$$

with

$$
E=A_{\mathrm{e} N}+B_{\mathrm{e} N} / Z_{\mathrm{c}}+C_{\mathrm{e} N} Z_{\mathrm{c}}+D_{\mathrm{e} N}
$$

where the subscripts $\mathcal{A}$ and $\mathcal{B}$ refer to the two port-related end planes of the calculated $N$-cell segment of a TL. In the same manner, the scattering matrix $\mathbf{S}^{[0]}$ of the $N$-cell odd-line can be computed.

We next consider the response of an $N$-cell CRLH coupler, which is schematically illustrated in Fig. 4(a). According to the coupled-mode analyses [49,51], the $2 \times 2$ scattering matrix $\mathbf{M}^{(\mathrm{c})} \equiv\left[M_{\uparrow \uparrow}^{(\mathrm{c})}, M_{\uparrow \downarrow}^{(\mathrm{c})} ; M_{\downarrow \uparrow}^{(\mathrm{c})}, M_{\downarrow \downarrow}^{(\mathrm{c})}\right]$ of a directional coupler can be deduced from the scattering matrices of the even/odd lines using the following formulas: 


$$
\begin{aligned}
M_{\uparrow \uparrow}^{(\mathrm{c})} & \equiv S_{\mathcal{A} \uparrow \rightarrow \mathcal{B} \uparrow}=\left(S_{\mathcal{B} \mathcal{A}}^{[\mathrm{e}]}+S_{\mathcal{B} \mathcal{A}}^{[\mathrm{o}]}\right) / 2, \\
M_{\downarrow \uparrow}^{(\mathrm{c})} & \equiv S_{\mathcal{A} \uparrow \rightarrow \mathcal{B} \downarrow}=\left(S_{\mathcal{B} \mathcal{A}}^{[\mathrm{e}]}-S_{\mathcal{B} \mathcal{A}}^{[\mathrm{o}]}\right) / 2, \\
M_{\uparrow \downarrow}^{(\mathrm{c})} & \equiv S_{\mathcal{A} \downarrow \rightarrow \mathcal{B} \uparrow}=M_{\downarrow \uparrow}^{(\mathrm{c})}, \\
M_{\downarrow \downarrow}^{(\mathrm{c})} & \equiv S_{\mathcal{A} \downarrow \rightarrow \mathcal{B} \downarrow}=M_{\uparrow \uparrow}^{(\mathrm{c})} .
\end{aligned}
$$

On the other hand, the self- and cross-reflections can also be obtained using the following expressions:

$$
\begin{aligned}
& \Gamma \equiv S_{\mathcal{A} \uparrow \rightarrow \mathcal{A} \uparrow}=S_{\mathcal{A} \downarrow \rightarrow \mathcal{A} \downarrow}=\left(S_{\mathcal{A} \mathcal{A}}^{[\mathrm{e}]}+S_{\mathcal{A} \mathcal{A}}^{[\mathrm{o}]}\right) / 2, \\
& \Upsilon \equiv S_{\mathcal{A} \uparrow \rightarrow \mathcal{A} \downarrow}=S_{\mathcal{A} \downarrow \rightarrow \mathcal{A} \uparrow}=\left(S_{\mathcal{A} \mathcal{A}}^{[\mathrm{e}]}-S_{\mathcal{A} \mathcal{A}}^{[\mathrm{o}]}\right) / 2 .
\end{aligned}
$$

In our design shown in Fig. 1(a), two 3-dB forward-wave couplers are required to realize the nonreciprocal transmission. To achieve the 3 -dB coupling, $\left|K_{0} l_{c}\right|$ needs to equal $\pi / 4$. Using the magnitude of coupling coefficient at $6 \mathrm{GHz}$ shown in Fig. 3(b), we obtain a coupling length $l_{\mathrm{c}}$ of $37 p$. Since $L_{\mathrm{m}}$-Off induces a stronger dispersion and causes a more severe restriction on the operating bandwidth, in what follows, we will focus on behaviors of the $3-\mathrm{dB}$ coupler with $L_{\mathrm{m}}$-Off. Using equations (20-25), we calculate the scattering parameters of the coupler at $L_{\mathrm{m}}$-Off with terminals of the corresponding uncoupled CRLH TL. The results are shown in Fig. 4(b), which indicate that the 3 - $\mathrm{dB}$ power splittings of the forward beams are achieved at $6 \mathrm{GHz}$. Meanwhile, the magnitudes of self- and crossreflections are very low at around $6 \mathrm{GHz}$. For comparison, the responses of $L_{\mathrm{m}}$-On are shown in Appendix C, which denote that the same 3 - $\mathrm{dB}$ power splittings are realized at $6 \mathrm{GHz}$ but with a weaker dispersion.

\section{B. Dynamic modulation of coupling coefficient}

To dynamically modulate the coupling coefficient, a radio-frequency (rf) superconducting quantum interference device (SQUID) is introduced between two coupled lines. As shown in Fig. 5(a), an rf-SQUID is coupled galvanically to right-handed segments of two CRLH TLs in each unit cell. We assume that in a unit cell, the contribution to $L_{\mathrm{R}}$ can be split into two parts: one is shared with the rf-SQUID loop and denoted $L_{\mathrm{S}}$; the left, including parasitic inductance, is depicted by $L_{\mathrm{P}}$, which means that $L_{\mathrm{R}}=L_{\mathrm{S}}+L_{\mathrm{P}}$. In our calculations, with $L_{\mathrm{R}}$ equal to $300 \mathrm{pH}$, a reasonable and achievable splitting is $\left\{L_{\mathrm{S}}, L_{\mathrm{P}}\right\}=\{200 \mathrm{pH}, 100 \mathrm{pH}\}$. Then, from the perspective of the rf-SQUID, the related superconducting loop has a geometric inductance $L_{\mathrm{G}}=2 L_{\mathrm{S}}+2 L_{\mathrm{B}}$, which is interrupted by a Josephson junction with critical current $I_{\mathrm{c}}$, as shown in the inset of Fig. 5(a). With these settings and definitions, we can express the effective SQUID inductance as

$$
L_{\mathrm{rf}}(\Phi)=L_{\mathrm{G}} \frac{1+\beta_{\mathrm{L}} \cos \left(2 \pi \Phi / \Phi_{0}\right)}{\beta_{\mathrm{L}} \cos \left(2 \pi \Phi / \Phi_{0}\right)}
$$

(a)

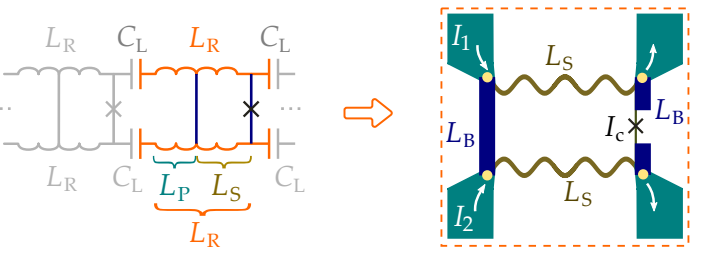

(b)

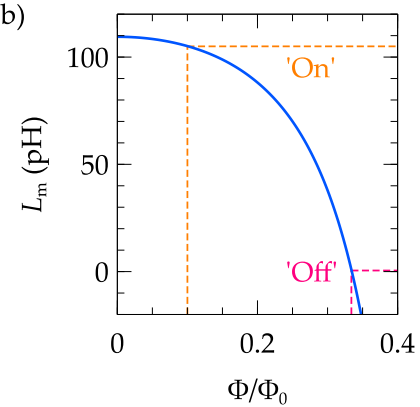

(c)

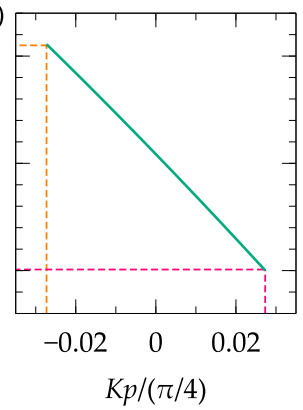

(d)

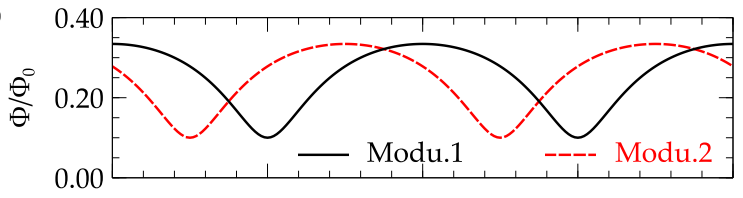

(e)

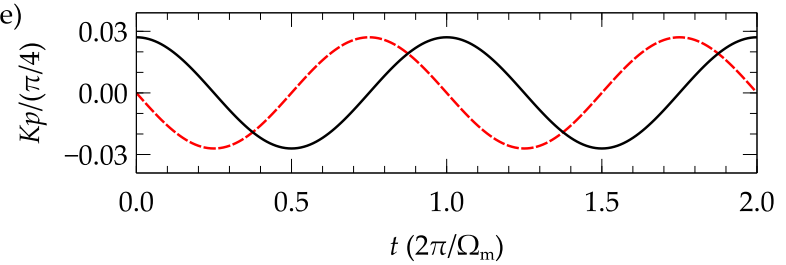

Figure 5. Coupling modulation via an rf-SQUID. (a) Circuit model for two coupled lines using an rf-SQUID. Only $L_{\mathrm{R}}$ and $C_{\mathrm{L}}$ of the coupled-line shown in Fig. 2(b) are displayed here for simplicity. The colored drawings illustrate a unit cell of the coupled-line. The right inset shows a possible design sketch. (b) The $\Phi$ dependence of $L_{\mathrm{m}}$. (c) Relationship of $K$ with $L_{\mathrm{m}}$. (d) Required time-varying $\Phi$ and (e) the corresponding modulated $K$ with two different initial phases of 0 and $\pi / 2$.

where $\Phi_{0}$ is the flux quantum, $\beta_{\mathrm{L}}=2 \pi I_{\mathrm{c}} L_{\mathrm{G}} / \Phi_{0}$ is the screening parameter, and $\Phi$ is the flux threading the SQUID loop. With these expressions and an assumption of $\beta_{\mathrm{L}}<1$, the effective mutual inductance can be estimated as

$$
L_{\mathrm{m}}(\Phi) \simeq L_{\mathrm{dir}}+L_{\mathrm{S}}^{2} / L_{\mathrm{rf}}(\Phi),
$$

where $L_{\text {dir }}$ expresses a flux-independent mutual inductance for direct inductive coupling between two lines, and the second term describes the effective second-order mutual inductance mediated by the SQUID $[52,53]$. For the setting of $\left\{L_{\mathrm{S}}, L_{\mathrm{B}}\right\}=\{200 \mathrm{pH}, 40 \mathrm{pH}\}$, a reasonable value of $70 \mathrm{pH}$ for $L_{\mathrm{dir}}$ can be achieved with a careful design. Then, by using Eqs. (26) and (27), $L_{\mathrm{m}}$ as a function of $\Phi$ is calculated with $\beta_{\mathrm{L}}=0.9$ (i.e., $I_{\mathrm{c}} \simeq 617 \mathrm{nA}$ ) and plotted in Fig. 5(b). Meanwhile, the $L_{\mathrm{m}}$ dependence of $K$ can be deduced from the results of the analyses described in 
Section III-B and is shown in Fig. 5(c). The connection between $K$ and $\Phi$ is made on the basis of Fig. 5(c) and Fig. 5(b), which implies that $K$ as a function of $\Phi$ is obtained. On the basis of such a connection, a temporal modulation of $K$ can be realized by applying time-varying $\Phi$, which is easy to implement with a local bias. Here, we only consider the modulation of $L_{\mathrm{m}}$ ranging from $L_{\mathrm{m}}$-Off to $L_{\mathrm{m}}$-On as marked in Fig. 5(b). Figures 5(d) and 5(e) show the required time-varying $\Phi$ and the corresponding $K$. In Fig. 5(e), the two modulation curves of $K$ versus time are just the required form in Eq. (1) with different initial phases of 0 and $\pi / 2$. As the explanation given in Appendix A, the required modulation frequency $\Omega_{\mathrm{m}}$ is about tens of megahertz, which is not a problem in the presence of local bias.

\section{NONRECIPROCAL RESPONSES}

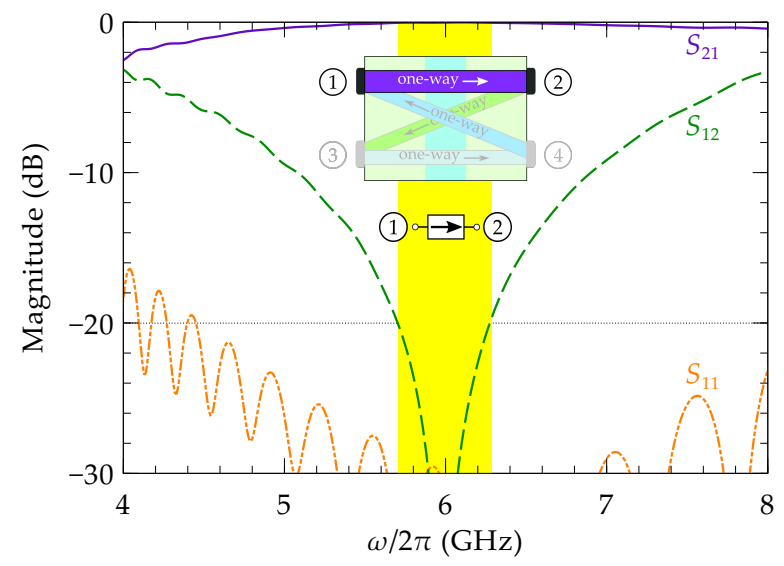

Figure 6. Nonreciprocal isolation response between port-1 and port-2 of the structure. A bandwidth of $580 \mathrm{MHz}$ can be achieved with the isolation over $20 \mathrm{~dB}$ at around $6 \mathrm{GHz}$, where the reflection is lower than $-29 \mathrm{~dB}$.

After we obtain the implementation of the modulated 3-dB coupler, the microwave responses of the entire structure with CRLH TLs can be deduced. Note that the scattering parameters for the coupler in Eqs. (20-23) are given under the condition without the modulation of the coupling coefficient. As described in Section II, when the coupling modulation expressed in Eq. (1) is applied, the scattering matrix $\mathbf{M}^{(\mathrm{c})}$ of the coupled-line is revised by the modulation initial phase $\phi$ as

$$
\mathbf{M}^{(\mathrm{c})}(\phi)=\left[\begin{array}{cc}
M_{\uparrow \uparrow}^{(\mathrm{c})} & \mathrm{e}^{-\mathrm{j} \phi} M_{\uparrow \downarrow}^{(\mathrm{c})} \\
\mathrm{e}^{\mathrm{j} \phi} M_{\downarrow \uparrow}^{(\mathrm{c})} & M_{\downarrow \downarrow}^{(\mathrm{c})}
\end{array}\right] .
$$

For stage-II, the two CRLH TLs are uncoupled but with different propagation lengths. The corresponding scattering matrix can be written as

$$
\mathbf{M}^{(\mathrm{b})}(\Delta \theta)=\left[\begin{array}{cc}
S_{\mathcal{C B}}^{[\uparrow]}\left(\theta_{0}+\Delta \theta\right) & 0 \\
0 & S_{\mathcal{C B}}^{[\downarrow]}\left(\theta_{0}\right)
\end{array}\right]
$$

where $\theta_{0}$ is trivial and denotes the common phase for the two CRLH TLs, which also refers to the operating frequency of $6 \mathrm{GHz}$. The scattering parameters $S_{\mathcal{C} \mathcal{B}}^{[\uparrow]}$ and $S_{\mathcal{C} \mathcal{B}}^{[\downarrow]}$ are calculated by the approach described in Section IV-A with both $L_{\mathrm{m}}$ and $C_{\mathrm{m}}$ equaling zero. By substituting expressions (28) and (29) into Eqs. (6) and (7), we can obtain the scattering matrices $\mathbf{M}^{(\Leftrightarrow)}$ and $\mathbf{M}^{(\Leftarrow)}$ for the entire structure based on CRLH TLs. Here, we redefine each element as

$$
\begin{gathered}
\mathbf{M}^{(\Rightarrow)} \equiv\left[\begin{array}{ll}
M_{\uparrow \uparrow}^{(\Rightarrow)} & M_{\uparrow \downarrow}^{(\Rightarrow)} \\
M_{\downarrow \uparrow}^{(\Rightarrow)} & M_{\downarrow \downarrow}^{(\Rightarrow)}
\end{array}\right]=\left[\begin{array}{ll}
M_{\mathcal{A} \uparrow \rightarrow \mathcal{D} \uparrow} & M_{\mathcal{A} \downarrow \rightarrow \mathcal{D} \uparrow} \\
M_{\mathcal{A} \uparrow \rightarrow \mathcal{D} \downarrow} & M_{\mathcal{A} \downarrow \rightarrow \mathcal{D} \downarrow}
\end{array}\right], \\
\mathbf{M}^{(\Leftarrow)} \equiv\left[\begin{array}{ll}
M_{\uparrow \uparrow}^{(\Leftarrow)} & M_{\uparrow \downarrow}^{(\Leftarrow)} \\
M_{\downarrow \uparrow}^{(\Leftarrow)} & M_{\downarrow \downarrow}^{(\Leftarrow)}
\end{array}\right]=\left[\begin{array}{ll}
M_{\mathcal{D} \uparrow \rightarrow \mathcal{A} \uparrow} & M_{\mathcal{D} \downarrow \rightarrow \mathcal{A} \uparrow} \\
M_{\mathcal{D} \uparrow \rightarrow \mathcal{A} \downarrow} & M_{\mathcal{D} \downarrow \rightarrow \mathcal{A} \downarrow}
\end{array}\right] .
\end{gathered}
$$

By using these redefined scattering parameters and labeling the four ports of the entire structure as port-1 to port-4 [see Fig. 1(a)], we can obtain the total scattering matrix as

$$
\mathbf{S}^{(\mathrm{tot})}=\left[\begin{array}{cccc}
\Gamma & M_{\uparrow \uparrow}^{(\Leftarrow)} & \Upsilon & M_{\uparrow \downarrow}^{(\Leftarrow)} \\
M_{\uparrow \uparrow}^{(\Rightarrow)} & \Gamma & M_{\uparrow \downarrow}^{(\Rightarrow)} & \Upsilon \\
\Upsilon & M_{\downarrow \uparrow}^{(\Leftarrow)} & \Gamma & M_{\downarrow \downarrow}^{(\Leftarrow)} \\
M_{\downarrow \uparrow}^{(\Rightarrow)} & \Upsilon & M_{\downarrow \downarrow}^{(\Rightarrow)} & \Gamma
\end{array}\right],
$$

where the self-reflection for each port and the crossreflection between ports located on the same side appear in the form of Eqs. (24) and (25), respectively. The reason for using $\Gamma$ and $\Upsilon$ as estimations for the reflections is that the cascade-induced inter-reflections are very small for waveguide-based structures. As a comparison, the exact total scattering matrix is deduced by a full-network analysis and shown in Appendix D.

From the results of the analyses in Section II, realizing nonreciprocity requires a specific setting on modulation phases in stage-I(-III) and phase shifts in stage-II. Here, $\left\{\phi_{1}, \Delta \theta, \phi_{2}\right\}=\{0, \pi / 2, \pi / 2\}$ is adopted again and substituted into equations (28) and (29). Note that, because there is dispersion of the whole system, all the settings of frequency-dependent parameters refer to our designed operation frequency of $6 \mathrm{GHz}$. Note also that $\Delta \theta$ equaling $\pi / 2$ requires 2.2 cells with current design parameters for the uncoupled CRLH TLs, but an integer multiple of cells can be achieved by slightly adjusting design parameters in stage-II. After some calculations, the total scattering parameters can be extracted from Eq. (32). Here, we 
(a)

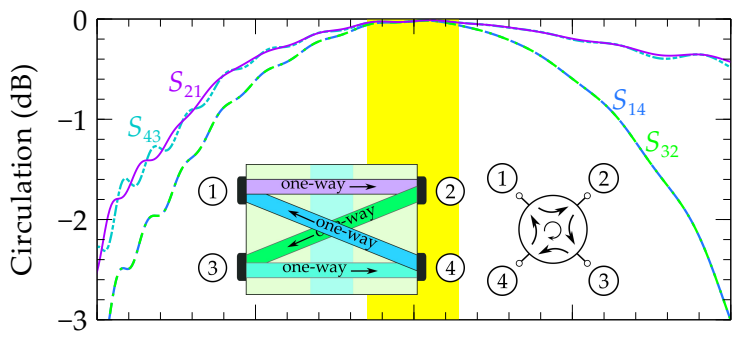

(b)

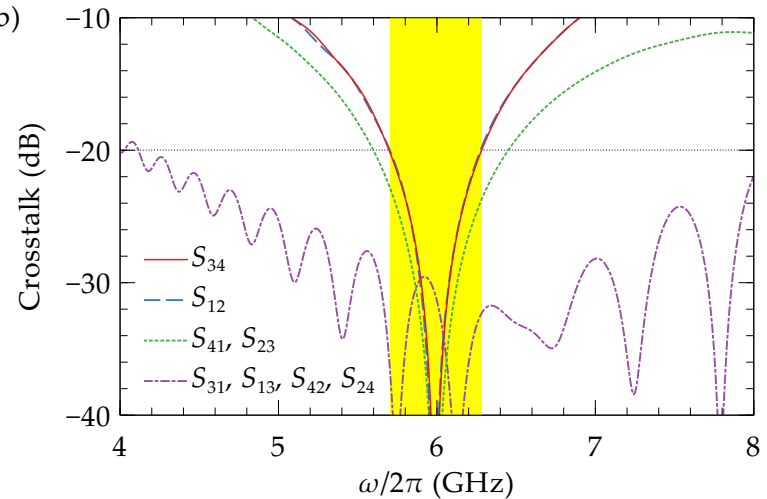

Figure 7. Nonreciprocal responses of (a) circulation and (b) crosstalk of the entire four-port structure. A bandwidth of $580 \mathrm{MHz}$ can be achieved with the isolation over $20 \mathrm{~dB}$ at around $6 \mathrm{GHz}$, where the crosstalks are lower than $-29 \mathrm{~dB}$.

demonstrate its nonreciprocal isolation between two ports and nonreciprocal circulations of four ports. To give a clear demonstration, a $2 \times 2$ scattering matrix related to port- 1 and port- 2 is extracted and defined as

$$
\mathbf{S}^{(1 \Leftrightarrow 2)} \equiv\left[\begin{array}{ll}
S_{11} & S_{12} \\
S_{21} & S_{22}
\end{array}\right]=\left[\begin{array}{cc}
\Gamma & M_{\uparrow \uparrow}^{(\Leftarrow)} \\
M_{\uparrow \uparrow}^{(\Rightarrow)} & \Gamma
\end{array}\right] .
$$

Figure 6 shows the scattering parameters between port1 and port-2. The results indicate that isolation over $20 \mathrm{~dB}$ for transmissions between port- 1 and port- 2 can be obtained with a bandwidth of $580 \mathrm{MHz}$ at around $6 \mathrm{GHz}$. Meanwhile, the absolute magnitudes of $S_{21}$ and $S_{11}$ show that the insertion loss and reflection are very small. These merits indicate that a well-performing isolator is realized by the designed structure with the CRLH TLs. Furthermore, the full scattering parameters for all four ports are displayed in Fig. 7. A clear nonreciprocal circulation from port-1 to port-4 is shown in Fig. 7(a) and all other crosstalk channels remain low-magnitude transmissions, as illustrated in Fig. 7(b). The results of Fig. 7 represent that a circulator can also be obtained with the same structure. It should be noted that, strictly speaking, this kind of circulator is not a common circulator, because a frequency shift of $\Omega_{\mathrm{m}}$ is introduced when waves are transmitted between ports attached to different TLs. Specifically, the frequency shift occurs in the transmission of $S_{32}$ and $S_{14}$, resulting from the interband transition between mode- $[\uparrow]$ and mode- $[\downarrow]$ induced by the dynamic modulation of the coupling coefficient (see Appendix A).
Such a nonreciprocal circulation can be classified as the nonreciprocity created in the frequency space [17, 36, 54]. A detailed interpretation is shown in Appendix A. However, such a nonreciprocal circulation can be used for applications concerned only with transfers of power.

\section{DISCUSSION}

To make the design into a reality from the circuit models, here, we propose to fabricate the CRLH TLs by a standard fabrication process for superconducting circuits. In general, to make the forward and backward waves see identical input impedance, a symmetric design of the unit cell of the coupled CRLH TLs is adopted and shown in Fig. 8(a) [compare with Fig. 2(b)]. By recalling the design parameters $\left\{L_{\mathrm{R}}, C_{\mathrm{R}}\right\}=\{300 \mathrm{pH}, 150 \mathrm{fF}\}$, these two contributions are realized with the common meander line and interdigital capacitor. To achieve an efficient transition between mode- $[\uparrow]$ and mode- $[\downarrow]$ under dynamic modulation, small $L_{\mathrm{R}}$-shifts can be implemented by slightly adjusting the width of meander lines (see Appendix A). The lefthanded elements of $\left\{L_{\mathrm{L}}, C_{\mathrm{L}}\right\}=\{1400 \mathrm{pH}, 560 \mathrm{fF}\}$ can be fabricated using a Josephson junction array and a parallel capacitor, in order to pursue a compact size and reduce the parasitic inductance and capacitance. Note that the parasitic inductance and capacitance should be considered and contributed to $\left\{L_{\mathrm{R}}, C_{\mathrm{R}}\right\}$. A careful design layout is needed to meet the required $C_{\mathrm{m}}=20 \mathrm{fF}$, and $L_{\mathrm{m}}=105 \mathrm{pH}$ for the $L_{\mathrm{m}}-\mathrm{On}$. The $L_{\mathrm{m}}$-Off and modulation of $L_{\mathrm{m}}$ are realized by constructing an rf-SQUID loop. Figure 8(b) displays a possible design layout for a unit cell, where the rf-SQUID loop is also outlined. A single local-bias line for all cells used for modulating the rf-SQUID loops can be designed and fabricated using airbridge technology [55,56], which is not illustrated in Fig. 8(b). The uncoupled CRLH TLs are easy to achieve by spatially separating the two TLs to cease their coupling. Importantly, the length of one unit cell less than $300 \mu \mathrm{m}$ is possible for these design parameters. Considering the cell numbers of 37 for each coupler in stage-I (stage-III) and (at least) 2 for the phase shift in stage-II, the estimated length for the entire structure is about $23 \mathrm{~mm}$, which is an acceptable size for a superconducting circuit chip. This means that a compact nonreciprocal isolator or circulator can be realized with our design on a microwave circuit chip.

In this work, we designed and demonstrated an isolator (circulator) with a bandwidth of $580 \mathrm{MHz}$ at around $6 \mathrm{GHz}$. However, these two metrics can be improved further by adjusting the design parameters. The operating frequency is readily changed by forming correspondingly responded couplers in stage-I (stage-III) and phase shifts in stage-II. A wider bandwidth is also possible; one solution is using a weaker dispersed coupled-line, but at a cost of the footprint of the entire structure. Another advanced approach is designing a super unit cell with a more linear dispersion curve (i.e., a smaller group-velocity dispersion), 
(a)

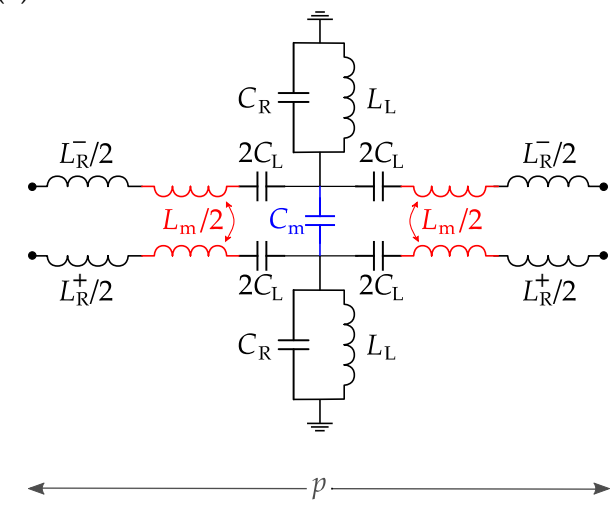

(b)

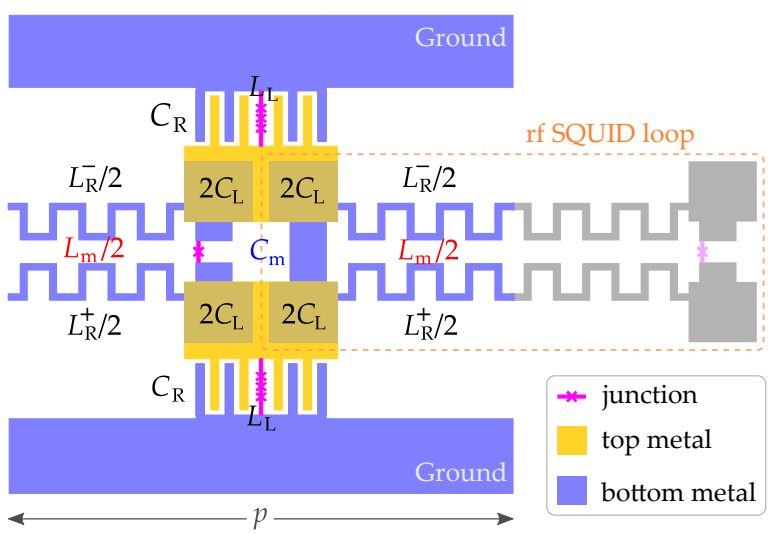

Figure 8. Physical implementation for the coupler design. (a) Circuit model for a symmetric unit cell of CRLH coupled-line. To achieve a slight asymmetry between two lines, small negative and positive $L_{\mathrm{R}}$-shifts are introduced in the upper and lower lines, respectively. (b) Design layout for a symmetric unit cell of CRLH coupled-line and the rf-SQUID loop for modulation of coupling coefficient.

where a delicate design is required [10, 57]. Moreover, the reflection (i.e., return loss) can be further reduced by adopting a tapered structure to more closely match the $50 \Omega$ line. Here, we demonstrated the design by the modulations of inductance or permeability in the couplers, but a similar functionality can also be implemented by modulating capacitance or permittivity in the other types of circuit $[1,58]$. As a spatiotemporal modulation-based nonreciprocal design, compared with the operating frequency, the modulation frequency is usually very low. Thus, the modulation wave can be simply filtered by spectral and spatial designs. Thus, in our scheme, in comparison with the general nonlinearity-based nonreciprocity, there is no strong microwave pumping, whose frequency is comparable to the operating frequency. This feature is also of benefit to its applications in quantum measurements.

\section{CONCLUSION}

In summary, we proposed a design of a magnetic-free broadband isolator or circulator with standard superconducting circuits. The designed structure consists of three stages, where two directional couplers are connected by inserted two uncoupled lines with different phase shifts. The two couplers require temporal modulations of the coupling coefficients with different initial phases. The entire structure can be fabricated using superconducting lines and Josephson junctions with a total line length of $23 \mathrm{~mm}$. In this work, the calculated results show that the nonreciprocal isolation over $20 \mathrm{~dB}$ with the bandwidth of $580 \mathrm{MHz}$ is realized at around the frequency of $6 \mathrm{GHz}$. Nonreciprocal circulations for microwave powers can also be realized with this structure. Moreover, these figures of merit can be further enhanced through the structural optimization design. Such an isolator or circulator will be very useful for cryogenic microwave connections or measurements in quantum integrated circuits.

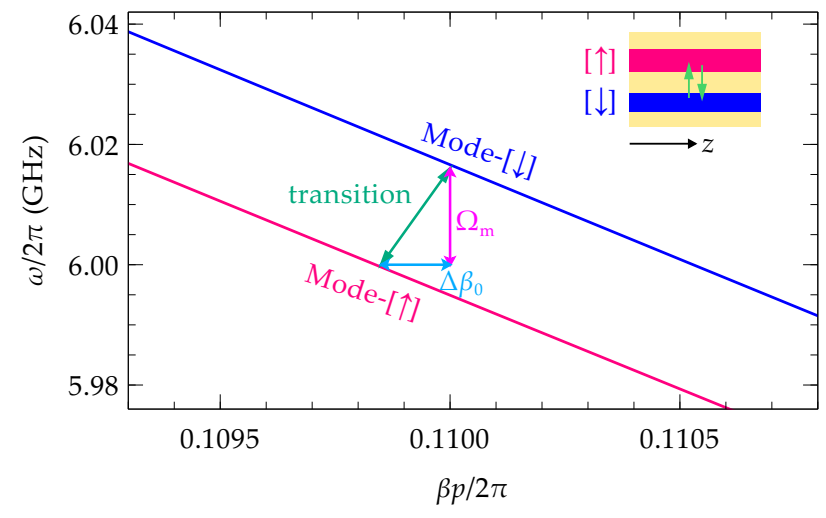

Figure 9. Interband transition or dynamical coupling between mode- $[\uparrow]$ and mode- $[\downarrow]$ of a dual-waveguide structure.

\section{ACKNOWLEDGMENTS}

The authors acknowledge useful discussions with Rui Wang and Iulia Zotova. This work was supported by JST [Moonshot R\&D][Grant Number JPMJMS2067] and CREST, JST (Grant No. JPMJCR1676). This article was partly based on results obtained from a project, JPNP16007, commissioned by the New Energy and Industrial Technology Development Organization (NEDO).

Note added.-Recently, we became aware of another work using a similar method to demonstrate travelingwave nonreciprocity [59].

\section{APPENDIX A: COUPLED-MODE THEORY}

We start by considering the photon transition process in a dual-waveguide system. The uncoupled dual-waveguide structure is represented by a permeability distribution 
$\mu_{\mathrm{s}}\left(\mathbf{r}_{\perp}\right)\left[\mathbf{r}_{\perp}=(x, y)\right]$, which is time-independent (i.e., static) and uniform along the $z$-direction (see the inset of Fig. 9). Such an uncoupled dual-waveguide structure possesses a band structure, as shown in Fig. 9, with uplocated and down-located modes corresponding to the first and second bands (i.e., mode- $[\uparrow]$ and mode- $[\downarrow]$ ), respectively. An interband transition between the two modes with frequencies and wave vectors, $\left(\omega_{\uparrow}, k_{\uparrow}\right),\left(\omega_{\downarrow}, k_{\downarrow}\right)$, can be induced by modulating the dual-waveguide structure with an additional time-varying relative permeability perturbation

$$
\mu\left(\mathbf{r}_{\perp}, t\right)=\mu_{\mathrm{s}}\left(\mathbf{r}_{\perp}\right)+\Delta \mu\left(\mathbf{r}_{\perp}, t\right)
$$

with

$$
\Delta \mu\left(\mathbf{r}_{\perp}, t\right)=\Delta \mu_{\mathrm{m}}\left(\mathbf{r}_{\perp}\right) \cos \left(\Omega_{\mathrm{m}} t+\phi\right),
$$

where $\Delta \mu_{\mathrm{m}}\left(\mathbf{r}_{\perp}\right)$ is the modulation amplitude distribution along the cross section, $\Omega_{\mathrm{m}}=\omega_{\downarrow}-\omega_{\uparrow}$ is the modulation frequency, and $\phi$ is the initial phase of the modulation profile [19, 41, 45].

In the modulated dual-waveguide structure, the magnetic field can be expressed as

$$
H\left(\mathbf{r}_{\perp}, z, t\right)=a_{\uparrow}(z) \psi_{\uparrow}\left(\mathbf{r}_{\perp}\right) \mathrm{e}^{\mathrm{j}\left(\omega_{\uparrow} t-\beta_{\uparrow} z\right)}+a_{\downarrow}(z) \psi_{\downarrow}\left(\mathbf{r}_{\perp}\right) \mathrm{e}^{\mathrm{j}\left(\omega_{\downarrow} t-\beta_{\downarrow} z\right)},
$$

where $\psi_{\uparrow, \downarrow}\left(\mathbf{r}_{\perp}\right)$ are the modal profiles and $a_{\uparrow, \downarrow}(z)$ denote the field amplitudes of mode-[个] and mode-[ $[\downarrow]$. For simplicity, we have assumed the quasi-TEM modes where the magnetic field has components majorly along the $\mathbf{r}_{\perp}$-direction. The magnetic field in Eq. (A3) satisfies Maxwell's wave equation,

$$
\nabla^{2} H\left(\mathbf{r}_{\perp}, z, t\right)=\frac{1}{c^{2}} \frac{\partial^{2}}{\partial t^{2}}\left[\mu\left(\mathbf{r}_{\perp}, t\right) H\left(\mathbf{r}_{\perp}, z, t\right)\right] .
$$

First, we consider the dual-waveguide structure without modulation (i.e., static). The magnetic fields satisfy the orthonormalization condition

$$
\frac{c^{2} \beta_{i}}{2 \omega_{i}^{2}} \int_{-\infty}^{\infty} \psi_{i}^{*}\left(\mathbf{r}_{\perp}\right) \psi_{j}\left(\mathbf{r}_{\perp}\right)=\delta_{i j} . \quad i, j=\uparrow, \downarrow
$$

With such a normalization, $\left|a_{i}\right|^{2}$ denotes the photon number flux carried by the mode- $[i](i=\uparrow, \downarrow)$. Then, we consider the modulated dual-waveguide structure. We substitute Eqs. (A1), (A2), and (A3) into Eq. (A4),

$$
\left(\nabla_{\perp}^{2}+\frac{\partial^{2}}{\partial z^{2}}\right) H\left(\mathbf{r}_{\perp}, z, t\right)=\frac{\mu_{\mathrm{s}}\left(\mathbf{r}_{\perp}\right)}{c^{2}} \frac{\partial^{2}}{\partial t^{2}} H\left(\mathbf{r}_{\perp}, z, t\right)+\frac{\Delta \mu_{\mathrm{m}}\left(\mathbf{r}_{\perp}\right)}{c^{2}} \frac{\partial^{2}}{\partial t^{2}}\left[\cos (\Omega t+\phi) H\left(\mathbf{r}_{\perp}, z, t\right)\right]
$$

Then we have

$$
\begin{aligned}
\mathrm{e}^{\mathrm{j}\left(\omega_{\uparrow} t-\beta_{\uparrow} z\right)}\left(-\mathrm{j} 2 \beta_{\uparrow} \psi_{\uparrow} \frac{\partial a_{\uparrow}}{\partial z}-a_{\uparrow} \beta_{\uparrow}^{2} \psi_{\uparrow}+a_{\uparrow} \nabla_{\perp}^{2} \psi_{\uparrow}\right)+\mathrm{e}^{\mathrm{j}\left(\omega_{\downarrow} t-\beta_{\downarrow} z\right)}\left(-\mathrm{j} 2 \beta_{\downarrow} \psi_{\downarrow} \frac{\partial a_{\downarrow}}{\partial z}-a_{\downarrow} \beta_{\downarrow}^{2} \psi_{\downarrow}+a_{\downarrow} \nabla_{\perp}^{2} \psi_{\downarrow}\right) \\
=\mathrm{e}^{\mathrm{j}\left(\omega_{\uparrow} t-\beta_{\uparrow} z\right)}\left(-\frac{\Delta \mu_{\mathrm{m}}}{2}\left[\left(\omega_{\uparrow}+\Omega_{\mathrm{m}}\right)^{2} \mathrm{e}^{\mathrm{j}\left(\Omega_{\mathrm{m}} t+\phi\right)}+\left(\omega_{\uparrow}-\Omega_{\mathrm{m}}\right)^{2} \mathrm{e}^{-\mathrm{j}\left(\Omega_{\mathrm{m}} t+\phi\right)}\right]-\omega_{\uparrow}^{2} \mu_{\mathrm{s}}\right) \frac{a_{\uparrow} \psi_{\uparrow}}{c^{2}} \\
\quad+\mathrm{e}^{\mathrm{j}\left(\omega_{\downarrow} t-\beta_{\downarrow} z\right)}\left(-\frac{\Delta \mu_{\mathrm{m}}}{2}\left[\left(\omega_{\downarrow}+\Omega_{\mathrm{m}}\right)^{2} \mathrm{e}^{\mathrm{j}\left(\Omega_{\mathrm{m}} t+\phi\right)}+\left(\omega_{\downarrow}-\Omega_{\mathrm{m}}\right)^{2} \mathrm{e}^{-\mathrm{j}\left(\Omega_{\mathrm{m}} t+\phi\right)}\right]-\omega_{\downarrow}^{2} \mu_{\mathrm{s}}\right) \frac{a_{\downarrow} \psi_{\downarrow}}{c^{2}},
\end{aligned}
$$

where we have used the slowly varying envelope approximation and dropped the terms $\partial^{2} a_{\uparrow, \downarrow} / \partial z^{2}$. Furthermore, on the basis of the definition of the modes in the unmodulated (i.e., static) waveguide and Eq. (A4), we obtain

$$
-\beta_{i}^{2} \psi_{i}+\nabla_{\perp}^{2} \psi_{i}=-\omega_{i}^{2} \mu_{\mathrm{s}} \frac{\psi_{i}}{c^{2}}, \quad i=\uparrow, \downarrow
$$

and by considering rotating wave approximation, we can derive the coupled-mode equation from Eq. (A7),

$$
\begin{aligned}
\mathrm{j} 2 \beta_{\uparrow} \psi_{\uparrow} & \frac{\partial a_{\uparrow}}{\partial z} \mathrm{e}^{\mathrm{j}\left(\omega_{\uparrow} t-\beta_{\uparrow} z\right)}+\mathrm{j} 2 \beta_{\downarrow} \psi_{\downarrow} \frac{\partial a_{\downarrow}}{\partial z} \mathrm{e}^{\mathrm{j}\left(\omega_{\downarrow} t-\beta_{\downarrow} z\right)} \\
& =\frac{\Delta \mu_{\mathrm{m}} \psi_{\uparrow}}{2 c^{2}}\left(\omega_{\uparrow}+\Omega_{\mathrm{m}}\right)^{2} a_{\uparrow} \mathrm{e}^{\mathrm{j}\left[\left(\omega_{\uparrow}+\Omega_{\mathrm{m}}\right) t-\beta_{\uparrow} z+\phi\right]}+\frac{\Delta \mu_{\mathrm{m}} \psi_{\downarrow}}{2 c^{2}}\left(\omega_{\downarrow}-\Omega_{\mathrm{m}}\right)^{2} a_{\downarrow} \mathrm{e}^{\mathrm{j}\left[\left(\omega_{\downarrow}-\Omega_{\mathrm{m}}\right) t-\beta_{\downarrow} z-\phi\right]}
\end{aligned}
$$

then, we have the equation of motion of the modulated dual-waveguide, 


$$
\mathrm{j} \frac{\partial}{\partial z}\left[\begin{array}{l}
a_{\uparrow} \\
a_{\downarrow}
\end{array}\right]=\left[\begin{array}{cc}
0 & K_{\uparrow \downarrow} \mathrm{e}^{-\mathrm{j}\left(\Delta \beta_{0} z+\phi\right)} \\
K_{\downarrow \uparrow} \mathrm{e}^{\mathrm{j}\left(\Delta \beta_{0} z+\phi\right)} & 0
\end{array}\right]\left[\begin{array}{l}
a_{\uparrow} \\
a_{\downarrow}
\end{array}\right],
$$

or, alternatively, the corresponding Hamiltonian can be given by

$$
\mathcal{H}=K_{\uparrow \downarrow} \mathrm{e}^{-\mathrm{j}\left(\Delta \beta_{0} z+\phi\right)} a_{\uparrow}^{\dagger} a_{\downarrow}+K_{\downarrow \uparrow} \mathrm{e}^{\mathrm{j}\left(\Delta \beta_{0} z+\phi\right)} a_{\uparrow} a_{\downarrow}^{\dagger},
$$

where $\Delta \beta_{0}=\beta_{\downarrow}-\beta_{\uparrow}$, and the coupling coefficient $K_{i j}(i \neq j)$ is calculated as

$$
K_{i j}=\frac{\omega_{i} \iint \mathrm{d} x \mathrm{~d} y \Delta \mu_{\mathrm{m}}\left(\mathbf{r}_{\perp}\right) \psi_{i}^{*}\left(\mathbf{r}_{\perp}\right) \psi_{j}\left(\mathbf{r}_{\perp}\right)}{4 c^{2} \beta_{i} \iint \mathrm{d} x \mathrm{~d} y \psi_{i}^{*}\left(\mathbf{r}_{\perp}\right) \psi_{i}\left(\mathbf{r}_{\perp}\right)},
$$

with the use of the orthonormalization condition of Eq. (A5), we obtain

$$
K_{\uparrow \downarrow}=K_{\downarrow \uparrow}^{*}=\frac{1}{8} \iint \mathrm{d} x \mathrm{~d} y \Delta \mu_{\mathrm{m}}\left(\mathbf{r}_{\perp}\right) \psi_{\uparrow}^{*}\left(\mathbf{r}_{\perp}\right) \psi_{\downarrow}\left(\mathbf{r}_{\perp}\right) .
$$

Generally, a pure real coupling coefficient can be obtained by the proper selection of initial phases of $\psi_{\uparrow}\left(\mathbf{r}_{\perp}\right)$ and $\psi_{\downarrow}\left(\mathbf{r}_{\perp}\right)$ in $(\mathrm{A} 13)$ and appears in the form

$$
K_{0} \equiv K_{\uparrow \downarrow}=K_{\downarrow \uparrow}
$$

The solution to Eq. (A10) is

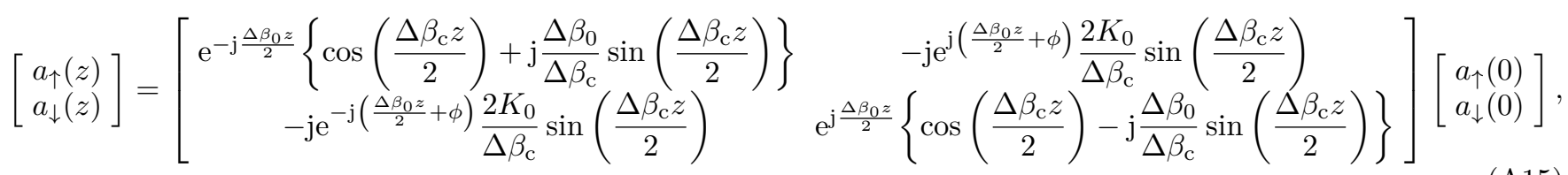

where

$$
\Delta \beta_{\mathrm{c}}=2 \sqrt{\left(\Delta \beta_{0} / 2\right)^{2}+K_{0}^{2}}
$$

By combining equations (A2), (A13), and (A14), we found that a time-varying coupling is generated by such a modulation of permeability and expressed as

$$
K(t)=K_{0} \cos \left(\Omega_{\mathrm{m}} t+\phi\right) .
$$

Phase-matching case. To achieve an efficient interband transition, the phase-matching condition should be satisfied. Thus, we can assume $\Delta \beta_{0}=0$, and Eq. (A10) is reduced to

$$
\mathrm{j} \frac{\partial}{\partial z}\left[\begin{array}{l}
a_{\uparrow} \\
a_{\downarrow}
\end{array}\right]=\left[\begin{array}{cc}
0 & K_{0} \mathrm{e}^{-\mathrm{j} \phi} \\
K_{0} \mathrm{e}^{\mathrm{j} \phi} & 0
\end{array}\right]\left[\begin{array}{l}
a_{\uparrow} \\
a_{\downarrow}
\end{array}\right],
$$

and the corresponding Hamiltonian can be expressed as

$$
\mathcal{H}=K_{0} \mathrm{e}^{-\mathrm{j} \phi} a_{\uparrow}^{\dagger} a_{\downarrow}+K_{0} \mathrm{e}^{\mathrm{j} \phi} a_{\uparrow} a_{\downarrow}^{\dagger}
$$

Correspondingly, the solution to Eq. (A18) is

$\left[\begin{array}{l}a_{\uparrow}(z) \\ a_{\downarrow}(z)\end{array}\right]=\left[\begin{array}{cc}\cos \left(K_{0} z\right) & -\mathrm{je}^{-\mathrm{j} \phi} \sin \left(K_{0} z\right) \\ -\mathrm{je}^{\mathrm{j} \phi} \sin \left(K_{0} z\right) & \cos \left(K_{0} z\right)\end{array}\right]\left[\begin{array}{c}a_{\uparrow}(0) \\ a_{\downarrow}(0)\end{array}\right]$,
Importantly, with Eq. (A16), there is $\Delta \beta_{\mathrm{c}}=2 K_{0}$, which corresponds to the wavenumber splitting of two normal modes induced by the coupling. When $\Omega_{\mathrm{m}}=\omega_{\downarrow}-\omega_{\uparrow}$ approaches zero (i.e., $\Omega_{\mathrm{m}} \ll \omega_{\downarrow}, \omega_{\uparrow}$ ), the two normal modes are known as the even-mode and odd-mode, so there is the expression

$$
K_{0}=\frac{\beta_{\text {even }}-\beta_{\text {odd }}}{2}
$$

where $\beta_{\text {even }}$ and $\beta_{\text {odd }}$ are propagation constants (i.e., wavenumbers) of the even and odd modes, respectively. For the dual-mode nonreciprocity, a more general description is presented in Ref. [46], where a useful insight is provided by a geometric picture.

In our design, to simultaneously realize the temporal modulation and efficient transition, a small frequency difference (i.e., $\Omega_{\mathrm{m}}$ ) between mode-[个] and mode- $[\downarrow]$ is required at $\Delta \beta_{0}=0$. In our implementation with superconducting circuit elements, this frequency difference can be readily realized by slightly tuning the design parameters. For example, at the operation frequency of $6 \mathrm{GHz}, \Omega_{\mathrm{m}}=20 \mathrm{MHz}$ is achieved by adding an $L_{\mathrm{R}}$-shift 
of -6 and $6 \mathrm{pH}$ for mode- $[\uparrow]$ and mode- $[\downarrow]$, respectively, as shown in Fig. 9. Notice that, when a different $L_{\mathrm{R}}$-shift is introduced to two waveguides, the coupled modes will not be perfect even and odd modes, which are called even-like and odd-like modes. However, these small shifts induce a negligible modification to the final results, since the magnitude of $6 \mathrm{pH}$ of the $L_{\mathrm{R}}$-shift is much smaller than that of $L_{\mathrm{R}}$ (equal to $300 \mathrm{pH}$ ). Accordingly, the reality of $\Omega_{\mathrm{m}} \ll \omega_{\downarrow}, \omega_{\uparrow}$ can also be found. A detailed discussion is provided in Appendix C.

For a CRLH TL, an equivalent relative permeability related to the material can be expressed in the form

$$
\mu=\frac{1}{\mu_{0}}\left(L_{\mathrm{R}}^{\prime}-\frac{1}{\omega^{2} C_{\mathrm{L}}^{\prime}}\right)
$$

where $\mu_{0}$ is the vacuum permeability, $L_{\mathrm{R}}^{\prime}$ is the per-unit length shunt inductance, and $C_{\mathrm{L}}^{\prime}$ is the times-unit length series capacitance of the CRLH TL [43]. Equation (A22) tells that $\mu$ can be tuned if the related $L_{\mathrm{R}}^{\prime}$ or $C_{\mathrm{L}}^{\prime}$ can be adjusted. Furthermore, the modulation of permeability in the form of (A2) is possible by the proper control of $L_{\mathrm{R}}^{\prime}$ or $C_{\mathrm{L}}^{\prime}$. In this work, we tune the mutual inductance $L_{\mathrm{m}}$ to realize the modulation. The reason is that $L_{\mathrm{m}}$ equivalently contributes to $L_{\mathrm{R}}$ for the even mode in the coupled-line [see Fig. 2(b)]. Alternatively, from the perspective of coupling coefficient, $K$ is modulated by varying $\beta_{\text {even }}$ according to equation (A21), which is essentially implemented by the modulation of $L_{\mathrm{m}}$.

\section{APPENDIX B: BASIC ANALYSES OF PERIODIC CRLH TLS}

Here, we give the basic analyses of a periodic CRLH TL as shown in Fig. 10(a) [see Fig. 2(a) for a unit cell] [43, 50]. By applying periodic boundary conditions (PBCs) related to the Bloch-Floquet theorem to the unit cell with length $p$, we can derive the CRLH TL propagation constant as

$$
\beta(\omega)=\frac{1}{p} \cos ^{-1}\left[1+\frac{Z(\omega) Y(\omega)}{2}\right]
$$

where the series impedance $(Z)$ and shunt admittance $(Y)$ of the CRLH TL unit cell are respectively expressed in the forms

$$
\begin{aligned}
& Z(\omega)=\mathrm{j}\left(\omega L_{\mathrm{R}}-\frac{1}{\omega C_{\mathrm{L}}}\right), \\
& Y(\omega)=\mathrm{j}\left(\omega C_{\mathrm{R}}-\frac{1}{\omega L_{\mathrm{L}}}\right) .
\end{aligned}
$$

Then, the characteristic impedance of the CRLH TL is given by

$$
Z_{0}(\omega)=\sqrt{Z(\omega) / Y(\omega)}
$$

Figure 10(b) displays the dispersion curve and characteristic impedance of a CRLH TL with the same $\left\{L_{\mathrm{R}}, C_{\mathrm{R}}\right\}$ and $\left\{L_{\mathrm{L}}, C_{\mathrm{L}}\right\}$ used in the main text. There are two important features. First, the propagation constant is about one order of magnitude greater than that of a common coplanar waveguide at around the frequency of $6 \mathrm{GHz}$. This means that the effective wavelength is roughly decreased by one order of magnitude, which implies that the structure size can be reduced significantly with CRLH TLs. Second, the characteristic impedance roughly equals $50 \Omega$ at around $6 \mathrm{GHz}$, which means that the impedance matching is not a critical problem with the CRLH TLs.

To calculate the transmissions of CRLH TLs, the $A B C D$-matrix of a symmetric unit cell is introduced and presented as follows:

$$
\left[\begin{array}{cc}
A_{\mathrm{u}} & B_{\mathrm{u}} \\
C_{\mathrm{u}} & D_{\mathrm{u}}
\end{array}\right]=\left[\begin{array}{cc}
1+Z Y / 2 & Z+Z^{2} Y / 4 \\
Y & 1+Z Y / 2
\end{array}\right] .
$$

For $N$ cells of the periodic CRLH TL, we have

$$
\left[\begin{array}{cc}
A_{\mathrm{u} N} & B_{\mathrm{u} N} \\
C_{\mathrm{u} N} & D_{\mathrm{u} N}
\end{array}\right]=\left[\begin{array}{cc}
A_{\mathrm{u}} & B_{\mathrm{u}} \\
C_{\mathrm{u}} & D_{\mathrm{u}}
\end{array}\right]^{N}
$$

Specifically, in the long-wavelength limit (i.e., $\beta p \ll 1$ ), Eq. (A23) is reduced to

$$
\beta(\omega)=-\mathrm{j} \sqrt{Z(\omega) Y(\omega)},
$$

which is known as the general expression of $\beta$ for a homogeneous TL shown in Fig. 10(a). Meanwhile, the $A B C D$ matrix of a homogeneous TL with length $l$ is given by

$$
\left[\begin{array}{ll}
A_{\mathrm{TL}} & B_{\mathrm{TL}} \\
C_{\mathrm{TL}} & D_{\mathrm{TL}}
\end{array}\right]=\left[\begin{array}{cc}
\cos (\beta l) & \mathrm{j} Z_{0} \sin (\beta l) \\
\mathrm{j} Z_{0}^{-1} \sin (\beta l) & \cos (\beta l)
\end{array}\right] .
$$

Once the $A B C D$-matrix for an $N$-cell network TL or homogeneous TL has been established, the corresponding scattering parameters $S_{i j}$ for terminations of impedance $Z_{\mathrm{c}}$ can be computed by using the well-known formula

$$
\left[\begin{array}{ll}
S_{11} & S_{12} \\
S_{21} & S_{22}
\end{array}\right]=\frac{1}{A+B / Z_{\mathrm{c}}+C Z_{\mathrm{c}}+D}\left[\begin{array}{cc}
A+B / Z_{\mathrm{c}}-C Z_{\mathrm{c}}-D & 2(A D-B C) \\
2 & -A+B / Z_{\mathrm{c}}-C Z_{\mathrm{c}}+D
\end{array}\right] .
$$


(a)

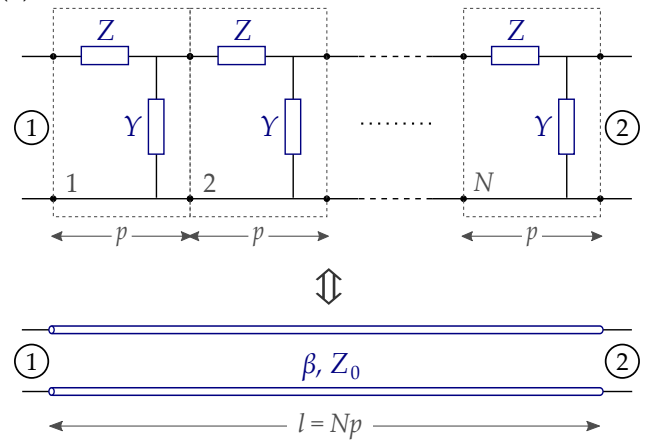

(b)

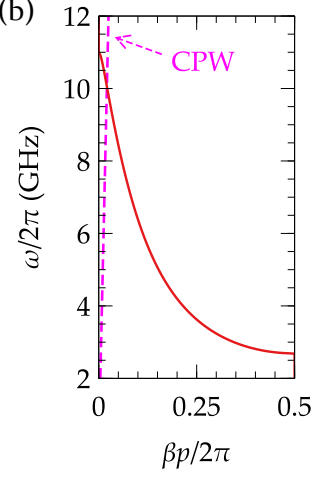

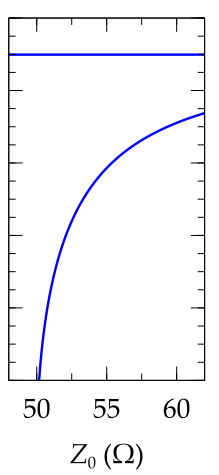

Figure 10. CRLH transmission line. (a) Periodic $N$-cell network and the equivalent homogeneous TL in the long-wavelength limit. (b) Frequency dependence of propagation constant (left) and characteristic impedance (right). (c) Transmissions and reflections of CRLH TL with length of $40 \mathrm{p}$ and terminal impedances of $50 \Omega$. The calculations are performed by two methods of $N$-cell network and homogeneous TL.

Here, for comparison, the transmission and reflection spectra are calculated by using both the $N$-cell network expression in (A28) and the homogeneous TL expression in (A30). The results are shown in Fig. 10(c) and indicate that there is no significant difference in the high-frequency region. Note that, from the dispersion curve shown in Fig. 10(b), the high-frequency region corresponds to the long-wavelength region, where the long-wavelength approximation is valid. From Fig. 10(c), it is found that the spectra obtained by two methods are matched well at around $6 \mathrm{GHz}$. Thus, a good analysis and prediction can be given by the coupled-mode theory for homogeneous TLs, then the accurate spectra can be obtained by using the $N$-cell network calculations.

\section{APPENDIX C: ASYMMETRICAL COUPLED-LINE}

As described Appendix A, to realize the temporal modulation and efficient transition between mode- $[\uparrow]$ and mode$[\downarrow]$, an asymmetric CRLH coupler is required in our design. The circuit model of a unit cell of the symmetric CRLH coupler and its equivalent even/odd line models are shown in Fig. 2(b) of the main text. However, it is not easy to obtain an equivalent circuit for an asymmetric CRLH coupler. Here, we analyze a homogeneous asymmetric coupled-line on the basis of the coupled-mode theory. In an asymmetric CRLH coupler, the normal modes become $c$ (even-like) and $\pi$ (odd-like) modes, which are not perfect even and odd modes [49, 60, 61]. The complex propagation constants $\gamma$ of the $c$ and $\pi$ modes are found to be

$$
\left(\begin{array}{cc}
a-\gamma^{2} & b \\
c & d-\gamma^{2}
\end{array}\right)\left(\begin{array}{l}
V_{\uparrow} \\
V_{\downarrow}
\end{array}\right)=0
$$

where

$$
\begin{array}{ll}
a=Z_{\uparrow} Y_{\uparrow}+Z_{\mathrm{m}} Y_{\mathrm{m}}, & b=Z_{\uparrow} Y_{\mathrm{m}}+Z_{\mathrm{m}} Y_{\downarrow} \\
c=Z_{\downarrow} Y_{\mathrm{m}}+Z_{\mathrm{m}} Y_{\uparrow}, & d=Z_{\downarrow} Y_{\downarrow}+Z_{\mathrm{m}} Y_{\mathrm{m}}
\end{array}
$$

with series impedances $Z_{\uparrow, \downarrow}$ and shunt admittances $Y_{\uparrow, \downarrow}$

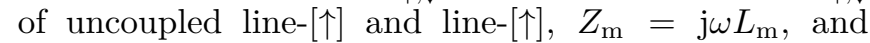
$Y_{\mathrm{m}}=-\mathrm{j} \omega C_{\mathrm{m}}$. Considering a lossless coupled-line, the propagation constants (i.e., wavenumbers) of the $c$ and $\pi$ modes are solved in the form

$$
\begin{aligned}
\beta_{c, \pi} & =-\mathrm{j} \gamma_{c, \pi} \\
& =-\mathrm{j}\left[\frac{a+d}{2} \pm \frac{1}{2} \sqrt{(a-d)^{2}+4 b c}\right]^{1 / 2} .
\end{aligned}
$$

Specifically, for even and odd modes, this expression is reduced to the identical form as Eq. (A29). Moreover, the uncoupled eigenmodes of the individual lines are obtained by setting $L_{\mathrm{m}}=C_{\mathrm{m}}=0$, and their isolated selfwavenumbers $\beta_{\uparrow}=-\mathrm{j} \sqrt{Z_{\uparrow} Y_{\uparrow}}$ and $\beta_{\downarrow}=-\mathrm{j} \sqrt{Z_{\downarrow} Y_{\downarrow}}$. Note that the self-wavenumbers for the individual lines in a coupled-line is not exactly equal to that of the uncoupled line, since the self-inductance and capacitance are modified in the presence of a neighboring line. However, these modifications are minute and omitted, especially for the consideration of the wavenumber difference $\Delta \beta_{0}=\beta_{\downarrow}-\beta_{\uparrow}$. Here, we assume that the asymmetry of the two lines is introduced by adding an $L_{\mathrm{R}}$-shift of -6 and $6 \mathrm{pH}$ for line- $[\uparrow]$ and line- $[\downarrow]$, respectively. The band structure of two uncoupled eigenmodes is just the plotting diagram shown in Fig. 9. In the asymmetric coupler, the $\Delta \beta_{\mathrm{c}}$ will be given by $\beta_{c}-\beta_{\pi}$, instead of $\beta_{\text {even }}-\beta_{\text {odd }}$. With the use of equation (A16), the coupling coefficient can be written as

$$
K_{0}=\frac{\sqrt{\Delta \beta_{\mathrm{c}}^{2}-\Delta \beta_{0}^{2}}}{2} .
$$


Now, we deduce the responses of the entire structure with asymmetric CRLH coupled-lines. Similarly to the treatment approach for the case with symmetric couplers in the main text: first, we assume that there is no modulation for the coupler (i.e., static) and obtain the required $l_{\mathrm{c}}$ and scattering matrix for the 3 -dB coupling at $6 \mathrm{GHz}$. Second, we consider the modulated coupler, where a modulation initial phase will be applied to the cross-coupling terms in the scattering matrix of the static coupler. Finally, the total responses of the entire structure can be obtained by a cascade multiplication of scattering matrices of stage-I to stage-III. Calculations of the second and third steps directly refer to the descriptions in Section $V$ of the main text. Here, we give the expressions of scattering parameters of a static asymmetric coupled line that appeared in the first step. Since there is no modulation, we can set $\Omega_{\mathrm{m}}=0$ and $\phi=0$ in Eq. (A15) and deduce the corresponding scattering parameters according to the definition of a scattering matrix. The scattering parameters of the asymmetric static coupled line are presented in the forms

$$
\begin{aligned}
& M_{\uparrow \uparrow}^{(\mathrm{c})} \equiv S_{\mathcal{A} \uparrow \rightarrow \mathcal{B} \uparrow}=\mathrm{e}^{-\mathrm{j} \frac{\Delta \beta_{0} l_{\mathrm{c}}}{2}}\left\{\cos \left(\frac{\Delta \beta_{\mathrm{c}} l_{\mathrm{c}}}{2}\right)+\mathrm{j} \frac{\Delta \beta_{0}}{\Delta \beta_{\mathrm{c}}} \sin \left(\frac{\Delta \beta_{\mathrm{c}} l_{\mathrm{c}}}{2}\right)\right\}, \\
& M_{\downarrow \uparrow}^{(\mathrm{c})} \equiv S_{\mathcal{A} \uparrow \rightarrow \mathcal{B} \downarrow}=-\mathrm{je}^{-\mathrm{j} \frac{\Delta \beta_{0} l_{\mathrm{c}}}{2}} \frac{2 K_{0}}{\Delta \beta_{\mathrm{c}}} \sin \left(\frac{\Delta \beta_{\mathrm{c}} l_{\mathrm{c}}}{2}\right), \\
& M_{\uparrow \downarrow}^{(\mathrm{c})} \equiv S_{\mathcal{A} \downarrow \rightarrow \mathcal{B} \uparrow}=-\mathrm{je}^{\mathrm{j} \frac{\Delta \beta_{0} l_{\mathrm{c}}}{2}} \frac{2 K_{0}}{\Delta \beta_{\mathrm{c}}} \sin \left(\frac{\Delta \beta_{\mathrm{c}} l_{\mathrm{c}}}{2}\right), \\
& M_{\downarrow \downarrow}^{(\mathrm{c})} \equiv S_{\mathcal{A} \downarrow \rightarrow \mathcal{B} \downarrow}=\mathrm{e}^{\mathrm{j} \frac{\Delta \beta_{0} l_{\mathrm{c}}}{2}}\left\{\cos \left(\frac{\Delta \beta_{\mathrm{c}} l_{\mathrm{c}}}{2}\right)-\mathrm{j} \frac{\Delta \beta_{0}}{\Delta \beta_{\mathrm{c}}} \sin \left(\frac{\Delta \beta_{\mathrm{c}} l_{\mathrm{c}}}{2}\right)\right\} .
\end{aligned}
$$

(a)

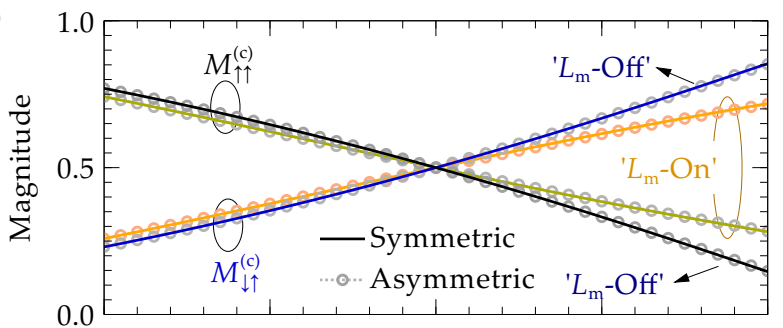

(b)

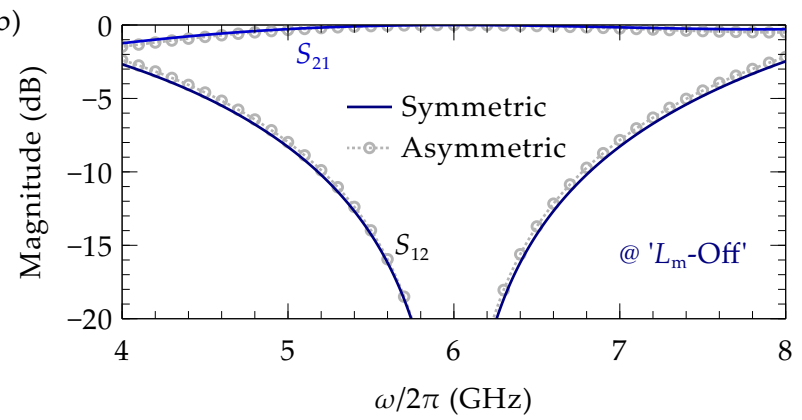

Figure 11. Responses using asymmetric and symmetric directional couplers. (a) Responses of $3-\mathrm{dB}$ coupler under the conditions of both $L_{\mathrm{m}}$-On and $L_{\mathrm{m}}$-Off. (b) Nonreciprocal isolation response under the condition of $L_{\mathrm{m}}$-Off.

By using Eqs. (A33-A38), the calculated scattering parameters are shown in Fig. 11(a) and depict the realization of 3-dB coupling. By substituting these results into the equations presented in Section V, we obtain the results of total scattering parameters displayed in Fig. 11(b). As a comparison, we also demonstrate the results of the symmetric coupled-line with the model of homogeneous TLs.
It is found that these differences are negligible, owing to the very small variation of the coupling coefficient induced by a small $L_{\mathrm{R}}$-shift (i.e., $\Delta \beta_{0} \ll K_{0}$ ). It should be noted that even an asymmetric coupled-line is used in the real design, but the interband transition effectively occurs at $\Delta \beta_{0} \approx 0$ under a dynamic modulation. The demonstration here using the scattering matrix of a static coupler with $\Omega_{\mathrm{m}}$ equal to 0 in the first step, provides an estimation (or a perspective) of the effect induced by a small asymmetry in the structure, but this is not the real process in the interband transition with $\Omega_{\mathrm{m}} \neq 0$.

\section{APPENDIX D: SCATTERING MATRIX OF INTERCONNECTED NETWORKS}

In the main text, we calculate the total responses with cascade multiplications of scattering matrices of stageI, stage-II, and stage-III. For each stage, the scattering matrix is expressed by a $2 \times 2$ matrix used for the twoport network. With such an expression, we have assumed that there are no reflections within TLs, so a physical four-port structure can be reduced to a two-port network. However, some weak inter-reflections may be induced by connections between neighboring stages, which are not considered in the cascade two-port network. To obtain an accurate response for the real full (four-port) network, we give a calculation approach with interconnected networks [49]. First, for each stage, we separate the four ports into the left-subgroup (including port-1 and port-2) and the right-subgroup (including port-3 and port-4), as shown in Fig. 12(a). Accordingly, the scattering matrix of stage-I 
can be expressed in the form

$$
\mathbf{S}^{(\mathrm{I})} \equiv\left[\begin{array}{cc}
{\left[\mathbf{S}_{\mathrm{LL}}^{(\mathrm{I})}\right]} & {\left[\mathbf{S}_{\mathrm{LR}}^{(\mathrm{I})}\right]} \\
{\left[\mathbf{S}_{\mathrm{RL}}^{(\mathrm{I})}\right]} & {\left[\mathbf{S}_{\mathrm{RR}}^{(\mathrm{I})}\right]}
\end{array}\right]=\left[\begin{array}{cc|cc}
\Gamma & \Upsilon & M_{\uparrow \uparrow}^{(\mathrm{c})} & \mathrm{e}^{-\mathrm{j} \phi_{1}} M_{\uparrow \downarrow}^{(\mathrm{c})} \\
\Upsilon & \Gamma & \mathrm{e}^{\mathrm{j} \phi_{1}} M_{\downarrow \uparrow}^{(\mathrm{c})} & M_{\downarrow \downarrow}^{(\mathrm{c})} \\
\hline M_{\uparrow \uparrow}^{(\mathrm{c})} & \mathrm{e}^{-\mathrm{j} \phi_{1}} M_{\uparrow \downarrow}^{(\mathrm{c})} & \Gamma & \Upsilon \\
\mathrm{e}^{\mathrm{j} \phi_{1}} M_{\downarrow \uparrow}^{(\mathrm{c})} & M_{\downarrow \downarrow}^{(\mathrm{c})} & \Upsilon & \Gamma
\end{array}\right],
$$

the scattering matrix for stage-II

$$
\mathbf{S}^{(\mathrm{II})} \equiv\left[\begin{array}{ll}
{\left[\mathbf{S}_{\mathrm{LL}}^{(\mathrm{II})}\right]} & {\left[\mathbf{S}_{\mathrm{LR}}^{(\mathrm{II})}\right]} \\
{\left[\mathbf{S}_{\mathrm{RL}}^{(\mathrm{II})}\right]} & {\left[\mathbf{S}_{\mathrm{RR}}^{(\mathrm{II})}\right]}
\end{array}\right]=\left[\begin{array}{cc|cc}
0 & 0 & S_{\mathcal{C B}}^{[\uparrow]} & 0 \\
0 & 0 & 0 & S_{\mathcal{C B}}^{[\downarrow]} \\
\hline S_{\mathcal{C B}}^{[\uparrow]} & 0 & 0 & 0 \\
0 & S_{\mathcal{C B}}^{[\downarrow]} & 0 & 0
\end{array}\right]
$$

and the scattering matrix for stage-III

$$
\mathbf{S}^{(\mathrm{III})} \equiv\left[\begin{array}{cc}
{\left[\mathbf{S}_{\mathrm{LL}}^{(\mathrm{III})}\right]} & {\left[\mathbf{S}_{\mathrm{LR}}^{(\mathrm{III})}\right]} \\
{\left[\mathbf{S}_{\mathrm{RL}}^{(\mathrm{III})}\right]} & {\left[\mathbf{S}_{\mathrm{RR}}^{(\mathrm{III})}\right]}
\end{array}\right]=\left[\begin{array}{cc|cc}
\Gamma & \Upsilon & M_{\uparrow \uparrow}^{(\mathrm{c})} & \mathrm{e}^{-\mathrm{j} \phi_{2}} M_{\uparrow \downarrow}^{(\mathrm{c})} \\
\Upsilon & \Gamma & \mathrm{e}^{\mathrm{j} \phi_{2}} M_{\downarrow \uparrow}^{(\mathrm{c})} & M_{\downarrow \downarrow}^{(\mathrm{c})} \\
\hline M_{\uparrow \uparrow}^{(\mathrm{c})} & \mathrm{e}^{-\mathrm{j} \phi_{2}} M_{\uparrow \downarrow}^{(\mathrm{c})} & \Gamma & \Upsilon \\
\mathrm{e}^{\mathrm{j} \phi_{2}} M_{\downarrow \uparrow}^{(\mathrm{c})} & M_{\downarrow \downarrow}^{(\mathrm{c})} & \Upsilon & \Gamma
\end{array}\right] .
$$

As the connection shown in Fig. 12(a), for two neighboring stages, the right-subgroup ports of the left-stage network are interconnected to the left-subgroup ports of the right-stage network. For such an interconnection, the scattering matrix for the connected network can be deduced recursively by

$$
\begin{aligned}
& \mathbf{S}_{\mathrm{LL}}^{(\imath \leftrightarrow \jmath)}=\mathbf{S}_{\mathrm{LL}}^{(\imath)}+\mathbf{S}_{\mathrm{LR}}^{(\imath)}\left(\mathbf{I}-\mathbf{S}_{\mathrm{LL}}^{(\jmath)} \mathbf{S}_{\mathrm{RR}}^{(\imath)}\right)^{-1} \mathbf{S}_{\mathrm{LL}}^{(\jmath)} \mathbf{S}_{\mathrm{RL}}^{(\imath)}, \\
& \mathbf{S}_{\mathrm{LR}}^{(\imath \leftrightarrow \jmath)}=\mathbf{S}_{\mathrm{LR}}^{(\imath)}\left(\mathbf{I}-\mathbf{S}_{\mathrm{LL}}^{(\jmath)} \mathbf{S}_{\mathrm{RR}}^{(\imath)}\right)^{-1} \mathbf{S}_{\mathrm{LR}}^{(\jmath)}, \\
& \mathbf{S}_{\mathrm{RL}}^{(\imath \leftrightarrow \jmath)}=\mathbf{S}_{\mathrm{RL}}^{(\jmath)}\left(\mathbf{I}-\mathbf{S}_{\mathrm{RR}}^{(\imath)} \mathbf{S}_{\mathrm{LL}}^{(\jmath)}\right)^{-1} \mathbf{S}_{\mathrm{RL}}^{(\imath)}, \\
& \mathbf{S}_{\mathrm{RR}}^{(\imath \leftrightarrow \jmath)}=\mathbf{S}_{\mathrm{RR}}^{(\jmath)}+\mathbf{S}_{\mathrm{RL}}^{(\jmath)}\left(\mathbf{I}-\mathbf{S}_{\mathrm{RR}}^{(\imath)} \mathbf{S}_{\mathrm{LL}}^{(\jmath)}\right)^{-1} \mathbf{S}_{\mathrm{RR}}^{(\imath)} \mathbf{S}_{\mathrm{LR}}^{(\jmath)},
\end{aligned}
$$

where the superscripts $\imath$ and $\jmath$ are the indices of connected left- and right-stage networks, respectively, $\mathbf{I}$ is a $2 \times 2$ identity matrix, and ()$^{-1}$ denotes the inverse of a matrix. By using these recurrence formulas of (A42), we can obtain the scattering matrix of the combined stages of I and II (i.e., I $\leftrightarrow$ II) as

$$
\mathbf{S}^{(\mathrm{I} \leftrightarrow \mathrm{II})} \equiv\left[\begin{array}{ll}
{\left[\mathbf{S}_{\mathrm{LL}}^{(\mathrm{I} \leftrightarrow \mathrm{II})}\right]} & {\left[\mathbf{S}_{\mathrm{LR}}^{(\mathrm{I} \leftrightarrow \mathrm{II})}\right]} \\
{\left[\mathbf{S}_{\mathrm{RL}}^{(\mathrm{I} \leftrightarrow \mathrm{II})}\right]} & {\left[\mathbf{S}_{\mathrm{RR}}^{(\mathrm{I} \leftrightarrow \mathrm{II})}\right]}
\end{array}\right]
$$

with settings $(\imath)=(\mathrm{I})$ and $(\jmath)=(\mathrm{II})$ in Eq. (A42) and substitutions of Eqs. (A39) and (A40). Furthermore, the scattering matrix for the entire structure (i.e., I $\leftrightarrow$ III) can be achieved,

$$
\mathbf{S}^{(\mathrm{I} \leftrightarrow \mathrm{III})} \equiv\left[\begin{array}{ll}
{\left[\mathbf{S}_{\mathrm{LL}}^{(\mathrm{I} \leftrightarrow \mathrm{III})}\right]} & {\left[\mathbf{S}_{\mathrm{LR}}^{(\mathrm{I} \leftrightarrow \mathrm{III})}\right]} \\
{\left[\mathbf{S}_{\mathrm{RL}}^{(\mathrm{I} \leftrightarrow \mathrm{III})}\right]} & {\left[\mathbf{S}_{\mathrm{RR}}^{(\mathrm{I} \leftrightarrow \mathrm{III})}\right]}
\end{array}\right]
$$

with settings $(\imath)=(\mathrm{I} \leftrightarrow \mathrm{II})$ and $(\jmath)=$ (III) in Eq. (A42) and substitutions of Eqs. (A43) and (A41). Finally, we rearrange the indices of port- 1 to port- 4 for the entire structure as that in the main text and provide a new notation for each scattering parameter (element) in expression (A44) as

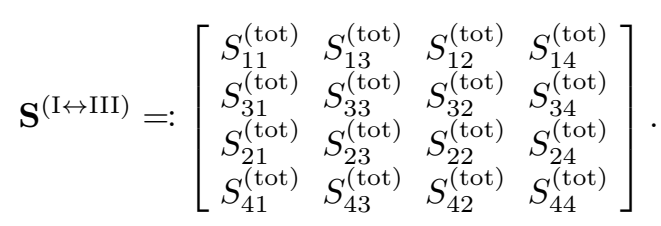

With such a full-network (four-port) method, the accurate total scattering parameters can be obtained. Figure 12(b) shows the calculated results, and the results of the cascade network (two-port) method are also demonstrated for comparison. It is found that the transmissions obtained by two methods match well. The reflections have some difference since the full network can reveal the inter-reflections between two connections. It is found that the magnitude of reflection from the full-network model is still less than $-20 \mathrm{~dB}$ within the frequency region of interest (around $6 \mathrm{GHz}$ ). 
(a)
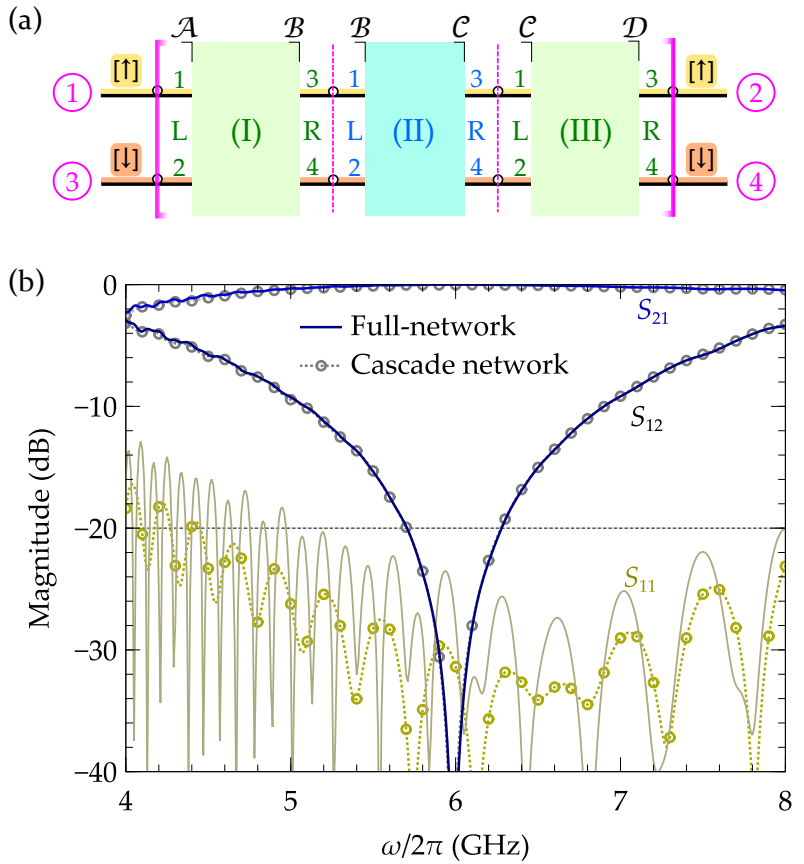

Figure 12. Results of full-network (four-port) and cascade network (two-port). (a) Schematic for full-network analysis. (b) Results of nonreciprocal isolation calculated by full-network and cascade network methods.

[1] Aravind Nagulu, Negar Reiskarimian, and Harish Krishnaswamy, "Non-reciprocal electronics based on temporal modulation," Nat. Electron. 3, 241-250 (2020).

[2] Ahmed Kord, Dimitrios L. Sounas, and Andrea Alù, "Microwave nonreciprocity," Proc. IEEE 108, 1728-1758 (2020).

[3] Dirk Jalas, Alexander Petrov, Manfred Eich, Wolfgang Freude, Shanhui Fan, Zongfu Yu, Roel Baets, Milos Popovic, Andrea Melloni, John D. Joannopoulos, et al., "What is - and what is not-an optical isolator," Nat. Photon. 7, 579-582 (2013).

[4] Leonardo Ranzani and Jose Aumentado, "Circulators at the quantum limit: Recent realizations of quantumlimited superconducting circulators and related approaches," IEEE Microw. Mag. 20, 112-122 (2019).

[5] A. Lupaşcu, S. Saito, T. Picot, P. C. de Groot, C. J. P. M. Harmans, and J. E. Mooij, "Quantum non-demolition measurement of a superconducting two-level system," Nat. Phys. 3, 119-123 (2007).

[6] François Mallet, Florian R. Ong, Agustin Palacios-Laloy, François Nguyen, Patrice Bertet, Denis Vion, and Daniel Esteve, "Single-shot qubit readout in circuit quantum electrodynamics," Nat. Phys. 5, 791-795 (2009).

[7] Evan Jeffrey, Daniel Sank, J. Y. Mutus, T. C. White, J. Kelly, R. Barends, Y. Chen, Z. Chen, B. Chiaro, A. Dunsworth, A. Megrant, P. J. J. O'Malley, C. Neill, P. Roushan, A. Vainsencher, J. Wenner, A. N. Cleland, and John M. Martinis, "Fast accurate state measurement with superconducting qubits," Phys. Rev. Lett. 112, 190504 (2014).
[8] I. Siddiqi, R. Vijay, F. Pierre, C. M. Wilson, M. Metcalfe, C. Rigetti, L. Frunzio, and M. H. Devoret, "RFdriven josephson bifurcation amplifier for quantum measurement," Phys. Rev. Lett. 93, 207002 (2004).

[9] T. Yamamoto, K. Inomata, M. Watanabe, K. Matsuba, T. Miyazaki, W. D. Oliver, Y. Nakamura, and J. S. Tsai, "Flux-driven Josephson parametric amplifier," Appl. Phys. Lett. 93, 042510 (2008).

[10] Kevin O'Brien, Chris Macklin, Irfan Siddiqi, and Xiang Zhang, "Resonant phase matching of Josephson junction traveling wave parametric amplifiers," Phys. Rev. Lett. 113, 157001 (2014).

[11] C. Macklin, K. O'Brien, D. Hover, M. E. Schwartz, V. Bolkhovsky, X. Zhang, W. D. Oliver, and I. Siddiqi, "A near-quantum-limited Josephson traveling-wave parametric amplifier," Science 350, 307-310 (2015).

[12] Archana Kamal, John Clarke, and M. H. Devoret, "Noiseless non-reciprocity in a parametric active device," Nat. Phys. 7, 311-315 (2011).

[13] Baleegh Abdo, Katrina Sliwa, Luigi Frunzio, and Michel Devoret, "Directional amplification with a Josephson circuit," Phys. Rev. X 3, 031001 (2013).

[14] A. Metelmann and A. A. Clerk, "Nonreciprocal photon transmission and amplification via reservoir engineering," Phys. Rev. X 5, 021025 (2015).

[15] Baleegh Abdo, Katrina Sliwa, S. Shankar, Michael Hatridge, Luigi Frunzio, Robert Schoelkopf, and Michel Devoret, "Josephson directional amplifier for quantum measurement of superconducting circuits," Phys. Rev. Lett. 112, 167701 (2014). 
[16] Hoi-Kwan Lau and Aashish A. Clerk, "Fundamental limits and non-reciprocal approaches in non-Hermitian quantum sensing," Nat. Commun. 9, 4320 (2018).

[17] F. Lecocq, L. Ranzani, G. A. Peterson, K. Cicak, R. W. Simmonds, J. D. Teufel, and J. Aumentado, "Nonreciprocal microwave signal processing with a field-programmable Josephson amplifier," Phys. Rev. Appl. 7, 024028 (2017).

[18] Baleegh Abdo, Nicholas T. Bronn, Oblesh Jinka, Salvatore Olivadese, Antonio D. Córcoles, Vivekananda P. Adiga, Markus Brink, Russell E. Lake, Xian Wu, David P. Pappas, and Jerry M. Chow, "Active protection of a superconducting qubit with an interferometric Josephson isolator," Nat. Commun. 10, 3154 (2019).

[19] Zongfu Yu and Shanhui Fan, "Complete optical isolation created by indirect interband photonic transitions," Nat. Photon. 3, 91-94 (2009).

[20] Giovanni Viola and David P. DiVincenzo, "Hall effect gyrators and circulators," Phys. Rev. X 4, 021019 (2014).

[21] Y. Shoji, K. Miura, and T. Mizumoto, "Optical nonreciprocal devices based on magneto-optical phase shift in silicon photonics," J. Opt. 18, 013001 (2016).

[22] Yi-Pu Wang, J. W. Rao, Y. Yang, Peng-Chao Xu, Y. S. Gui, B. M. Yao, J. Q. You, and C.-M. Hu, "Nonreciprocity and unidirectional invisibility in cavity magnonics," Phys. Rev. Lett. 123, 127202 (2019).

[23] Yu Shi, Zongfu Yu, and Shanhui Fan, "Limitations of nonlinear optical isolators due to dynamic reciprocity," Nat. Photon. 9, 388-392 (2015).

[24] Leonardo Del Bino, Jonathan M. Silver, Michael T. M. Woodley, Sarah L. Stebbings, Xin Zhao, and Pascal Del'Haye, "Microresonator isolators and circulators based on the intrinsic nonreciprocity of the Kerr effect," Optica 5, 279-282 (2018).

[25] Andrés Rosario Hamann, Clemens Müller, Markus Jerger, Maximilian Zanner, Joshua Combes, Mikhail Pletyukhov, Martin Weides, Thomas M. Stace, and Arkady Fedorov, "Nonreciprocity realized with quantum nonlinearity," Phys. Rev. Lett. 121, 123601 (2018).

[26] Dimitrios L. Sounas and Andrea Alù, "Non-reciprocal photonics based on time modulation," Nat. Photon. 11, 774 (2017).

[27] Negar Reiskarimian and Harish Krishnaswamy, "Magnetic-free non-reciprocity based on staggered commutation," Nat. Commun. 7, 11217 (2016).

[28] Eric I. Rosenthal, Benjamin J. Chapman, Andrew P. Higginbotham, Joseph Kerckhoff, and K. W. Lehnert, "Breaking Lorentz reciprocity with frequency conversion and delay," Phys. Rev. Lett. 119, 147703 (2017).

[29] Nima Chamanara, Sajjad Taravati, Zoé-Lise Deck-Léger, and Christophe Caloz, "Optical isolation based on spacetime engineered asymmetric photonic band gaps," Phys. Rev. B 96, 155409 (2017).

[30] Yao Yu, Giuseppe Michetti, Ahmed Kord, Michele Pirro, Dimitrios L. Sounas, Zhicheng Xiao, Cristian Cassella, Andrea Alu, and Matteo Rinaldi, "Highly-linear magnet-free microelectromechanical circulators," J. Microelectromech. Syst. 28, 933-940 (2019).

[31] L. D. Tóth, N. R. Bernier, A. Nunnenkamp, A. K. Feofanov, and T. J. Kippenberg, "A dissipative quantum reservoir for microwave light using a mechanical oscillator," Nat. Phys. 13, 787-793 (2017).

[32] Zhen Shen, Yan-Lei Zhang, Yuan Chen, Fang-Wen Sun, Xu-Bo Zou, Guang-Can Guo, Chang-Ling Zou, and ChunHua Dong, "Reconfigurable optomechanical circulator and directional amplifier," Nat. Commun. 9, 1797 (2018).

[33] S. Barzanjeh, M. Wulf, M. Peruzzo, M. Kalaee, P. B. Dieterle, O. Painter, and J. M. Fink, "Mechanical onchip microwave circulator," Nat. Commun. 8, 953 (2017).

[34] G. A. Peterson, F. Lecocq, K. Cicak, R. W. Simmonds, J. Aumentado, and J. D. Teufel, "Demonstration of efficient nonreciprocity in a microwave optomechanical circuit," Phys. Rev. X 7, 031001 (2017).

[35] Hugo Lira, Zongfu Yu, Shanhui Fan, and Michal Lipson, "Electrically driven nonreciprocity induced by interband photonic transition on a silicon chip," Phys. Rev. Lett. 109, 033901 (2012).

[36] K. M. Sliwa, M. Hatridge, A. Narla, S. Shankar, L. Frunzio, R. J. Schoelkopf, and M. H. Devoret, "Reconfigurable Josephson circulator/directional amplifier," Phys. Rev. X 5, 041020 (2015).

[37] Nicholas A. Estep, Dimitrios L. Sounas, Jason Soric, and Andrea Alù, "Magnetic-free non-reciprocity and isolation based on parametrically modulated coupled-resonator loops," Nat. Phys. 10, 923-927 (2014).

[38] Benjamin J. Chapman, Eric I. Rosenthal, Joseph Kerckhoff, Bradley A. Moores, Leila R. Vale, J. A. B. Mates, Gene C. Hilton, Kevin Lalumière, Alexandre Blais, and K. W. Lehnert, "Widely tunable on-chip microwave circulator for superconducting quantum circuits," Phys. Rev. X 7, 041043 (2017).

[39] Youngsun Choi, Choloong Hahn, Jae Woong Yoon, Seok Ho Song, and Pierre Berini, "Extremely broadband, on-chip optical nonreciprocity enabled by mimicking nonlinear anti-adiabatic quantum jumps near exceptional points," Nat. Commun. 8, 14154 (2017).

[40] Leonardo Ranzani, Shlomi Kotler, Adam J. Sirois, Michael P. DeFeo, Manuel Castellanos-Beltran, Katarina Cicak, Leila R. Vale, and José Aumentado, "Wideband isolation by frequency conversion in a Josephson-junction transmission line," Phys. Rev. Applied 8, 054035 (2017).

[41] Kejie Fang, Zongfu Yu, and Shanhui Fan, "Photonic Aharonov-Bohm effect based on dynamic modulation," Phys. Rev. Lett. 108, 153901 (2012).

[42] Lawrence D. Tzuang, Kejie Fang, Paulo Nussenzveig, Shanhui Fan, and Michal Lipson, "Non-reciprocal phase shift induced by an effective magnetic flux for light," Nat. Photon. 8, 701-705 (2014).

[43] A. Lai, C. Caloz, and T. Itoh, "Composite right/lefthanded transmission line metamaterials," IEEE Microw. Mag. 5, 34-50 (2004).

[44] Akimichi Hirota, Yukihiro Tahara, and Naofumi Yoneda, "A compact forward coupler using coupled composite right/left-handed transmission lines," IEEE Trans. Microw. Theory Tech. 57, 3127-3133 (2009).

[45] Chengzhi Qin, Feng Zhou, Yugui Peng, Dimitrios Sounas, Xuefeng Zhu, Bing Wang, Jianji Dong, Xinliang Zhang, Andrea Alù, and Peixiang Lu, "Spectrum control through discrete frequency diffraction in the presence of photonic gauge potentials," Phys. Rev. Lett. 120, 133901 (2018).

[46] Leonardo Ranzani and José Aumentado, "A geometric description of nonreciprocity in coupled two-mode systems," New J. Phys. 16, 103027 (2014).

[47] Bernard Yurke, Samuel L. McCall, and John R. Klauder, "SU(2) and SU(1,1) Interferometers," Phys. Rev. A 33, 4033-4054 (1986).

[48] Ulf Leonhardt, Essential Quantum Optics: From Quantum Measurements to Black Holes (Cambridge University Press, 2010). 
[49] Rajesh K Mongia, J Hong, Prakash Bhartia, and Inder Jit Bahl, RF and Microwave Coupled-Line Circuits (Artech house, 2007).

[50] Ferran Martin, Artificial Transmission Lines for RF and Microwave Applications (John Wiley \& Sons, 2015).

[51] C. Caloz, A. Sanada, and T. Itoh, "A novel composite right-/left-handed coupled-line directional coupler with arbitrary coupling level and broad bandwidth," IEEE Trans. Microw. Theory Tech. 52, 980-992 (2004).

[52] Borja Peropadre, David Zueco, Friedrich Wulschner, Frank Deppe, Achim Marx, Rudolf Gross, and Juan José García-Ripoll, "Tunable coupling engineering between superconducting resonators: From sidebands to effective gauge fields," Phys. Rev. B 87, 134504 (2013).

[53] Friedrich Wulschner, Jan Goetz, Fabian R Koessel, Elisabeth Hoffmann, Alexander Baust, Peter Eder, Michael Fischer, Max Haeberlein, Manuel J Schwarz, Matthias Pernpeintner, Edwar Xie, Ling Zhong, Christoph W Zollitsch, Borja Peropadre, Juan-Jose Garcia Ripoll, Enrique Solano, Kirill G Fedorov, Edwin P Menzel, Frank Deppe, Achim Marx, and Rudolf Gross, "Tunable coupling of transmission-line microwave resonators mediated by an $\mathrm{rf}$ SQUID," EPJ Quantum Tech. 3, 1 (2016).

[54] Kamal Abdelsalam, Tengfei Li, Jacob B. Khurgin, and Sasan Fathpour, "Linear isolators using wavelength conversion," Optica 7, 209 (2020).

[55] Zijun Chen, A. Megrant, J. Kelly, R. Barends, J. Bochmann, Yu Chen, B. Chiaro, A. Dunsworth, E. Jeffrey, J. Y. Mutus, P. J. J. O'Malley, C. Neill, P. Roushan, D. Sank, A. Vainsencher, J. Wenner, T. C. White, A. N. Cleland, and John M. Martinis, "Fabrication and char- acterization of aluminum airbridges for superconducting microwave circuits," Appl. Phys. Lett. 104, 052602 (2014).

[56] Hiroto Mukai, Keiichi Sakata, Simon J Devitt, Rui Wang, Yu Zhou, Yukito Nakajima, and Jaw-Shen Tsai, "Pseudo$2 d$ superconducting quantum computing circuit for the surface code: proposal and preliminary tests," New J. Phys. 22, 043013 (2020).

[57] Luca Planat, Arpit Ranadive, Rémy Dassonneville, Javier Puertas Martínez, Sébastien Léger, Cécile Naud, Olivier Buisson, Wiebke Hasch-Guichard, Denis M. Basko, and Nicolas Roch, "Photonic-crystal Josephson traveling-wave parametric amplifier," Phys. Rev. X 10, 021021 (2020).

[58] Tolga Dinc, Mykhailo Tymchenko, Aravind Nagulu, Dimitrios Sounas, Andrea Alu, and Harish Krishnaswamy, "Synchronized conductivity modulation to realize broadband lossless magnetic-free non-reciprocity," Nat. Commun. 8, 795 (2017).

[59] Mahdi Naghiloo, Kaidong Peng, Yufeng Ye, Gregory Cunningham, and Kevin P. O'Brien, "Broadband microwave isolation with adiabatic mode conversion in coupled superconducting transmission lines," (2021), arXiv:2103.07793 [quant-ph].

[60] V.K. Tripathi, "Asymmetric coupled transmission lines in an inhomogeneous medium," IEEE Trans. Microw. Theory Tech. 23, 734-739 (1975).

[61] R. Islam and G.V. Eleftheriades, "Printed high-directivity metamaterial MS/NRI coupled-line coupler for signal monitoring applications," IEEE Microw. Wireless Compon. Lett. 16, 164-166 (2006). 